\title{
Comparative studies on enantioseparation of New Psychoactive Substances using cyclodextrin-assisted capillary electrophoresis with UV detection
}

\author{
Eva-Maria Hubner $^{1}$ Patrick Steinkellner $^{1}$ Martin G. Schmid ${ }^{1 *}$ \\ ${ }^{1}$ Department of Pharmaceutical Chemistry, Institute of Pharmaceutical Sciences, University of Graz, Austria
}

\section{Check for updates}

Correspondence to: Martin G. Schmid, Department of Pharmaceutical Chemistry, Institute of Pharmaceutical Sciences, University of Graz, Schubertstra $\beta$ e 1,8010 Graz, Austria;

Email: martin.schmid@uni-graz.at

Received: December 23, 2021;

Accepted: January 22, 2022;

Published: January 25, 2022.

Citation: Hubner E, Steinkellner P and Schmid MG. Comparative studies on enantioseparation of New Psychoactive Substances using cyclodextrin-assisted capillary electrophoresis with UV detection. $J$ Pharm Biopharm Res, 2022, 3(1): 187-205.

https://doi.org/10.25082/JPBR.2021.01.004

Copyright: () 2022 Eva-Maria Hubner et al. This is an open access article distributed under the terms of the Creative Commons Attribution License, which permits unrestricted use, distribution, and reproduction in any medium, provided the original author and source are credited.

\begin{abstract}
New psychoactive substances (NPS) count as psychoactive substances, which are slightly modified compared to illicit drugs regarding their chemical structure to circumvent law. Compared to classical drugs such as heroin, cocaine, or amphetamine, they show similar psychoactive effects, however, because of their novelty there is few knowledge about their side effects or toxicity. NPS are available as different chemical substance classes, among them chiral novel derivatives of amphetamine, cathinone, and ketamine. Since in most cases no clinical studies are available about the possibly different effects of the two enantiomers, there is a big demand for enantioseparation method development. Besides high-performance separation techniques such as gas chromatography or HPLC, capillary electrophoresis has turned out to be a powerful alternative for chiral separation development. The addition of chiral additives such as cyclodextrins to the background electrolyte often results in successful attempts. The present study compares the chiral separation power of different previously used non-charged $\beta$-cyclodextrins, among them native $\beta$-cyclodextrin as well as some of its derivatives such as acetyl-, and 2-hydroxypropyl- $\beta$-cyclodextrin, with the negatively charged derivatives carboxymethyl-, carboxyethyl- and succinyl- $\beta$-cyclodextrin by capillary zone electrophoresis. A total of 136 chiral NPS were investigated with these cyclodextrins, 122 of them were resolved in their enantiomers successfully by means of a simple electrolyte composition consisting of $10 \mathrm{mM}$ aqueous sodium hydrogen phosphate buffer, $\mathrm{pH} 2.5$ and $10 \mathrm{mM}$ of the chiral selector. Furthermore, the presented method turned out to be useful to distinguish between positional isomers and examples for both enantiomer order and positional order for seized samples are given.
\end{abstract}

Keywords: native $\beta$-cyclodextrin, acetyl- $\beta$-cyclodextrin, 2 -hydroxypropyl- $\beta$-cyclodextrin, carboxymethyl- $\beta$-cyclodextrin, carboxyethyl- $\beta$-cyclodextrin, succinyl- $\beta$-cyclodextrin, novel psychoactive substances, chiral

\section{Introduction}

Besides classic illicit drugs such as cannabis, amphetamine, cocaine or heroin, the so-called New Psychoactive Substances (NPS) came up in generations since about two decades [1] because of high prices along with poor quality of certain illicit drugs at that time. Minor structural changes in the chemical structure of several classic drugs resulted in an emerge of a huge number of novel derivatives. The main intention was to circumvent law against their abuse and additionally to compete price and quality. Since the middle of the 2000s [2] the worldwide drug market was flooded with such designer drugs with aim to mimic the psychoactive effects of classic drugs and to avoid violation of drug laws.

The United Nations Single Convention on Narcotic Drugs of 1961 provided a base for the specific regulation for production, use, possession, and distribution of illicit drugs [3]. Herein, narcotic plants, substances and preparations are listed, for example cannabis, opium, cocaine, methadone, and heroin [4]. In 1972, psychotropics such as amphetamine (Speed), methamphetamine (Crystal meth), LSD and MDMA (Ecstasy) were included [5]. This law also comprises derivatives like isomers, salts, esters, and ethers of the listed substances. Although a big variety of these compounds is covered by this convention and the corresponding national laws, drug chemists found creative attempts to produce and trade legal alternatives. For example, structural alterations such as introduction of halogen substituents, carbonyl-, or carboxy-groups led to new derivatives with similar psychoactive effects. Contrary to their parent structures, they were and are partially still legal. Marketed as "bath salts", "bird cage cleaners", "research chemicals" these so-called "legal highs" are distributed mostly over the internet. The distribution 
of drugs, not solely via the dark net, is a concerning trend that rose to a new peak during the ongoing pandemic [2]. Figure 1 shows offers of cathinones as crystals or tablets available in the clearnet.

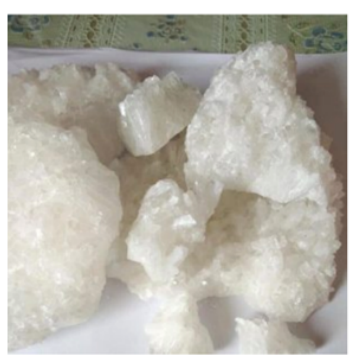

3-CEC Crystals

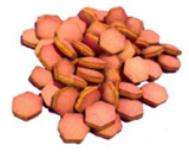

3 CMC Pellets $180 \mathrm{mg}$

Figure 1 Cathinone crystals and tablets offered in an online shop

According to the latest European Drug Report currently 830 NPS are monitored by the European Monitoring Centre for Drugs and Drug Addition (EMCDDA), 46 of them were reported for the first time in 2020. Though the number of newly reported substance is declining in the last years, it is still on a high level [2].

Many of these novel designer drugs contain an asymmetric centre with the consequence that two enantiomers are possible. In general, chiral substances might exhibit different pharmacological characteristics for each enantiomer as pharmaceutical drug substances do. It is already well-known that the psychoactive effect of some classic chiral addictive substances is restricted mainly to one distinct enantiomer, as it applies e. g. for S-(+)-amphetamine, S-(+)methamphetamine or S-(+)-ketamine [6-9]. This fact leads to the conclusion that derivatives of the afore mentioned compound classes might exhibit pharmacological differences with respect to their enantiomers as well, however, besides some exceptions [10-15], there is little or no knowledge about it.

Therefore, the development of chiral separation techniques for chiral NPS is of huge interest. It serves to determine the chiral status of samples as well as to allocate positional isomers. Furthermore, changes within the illegal drug market can be monitored more precisely. The knowledge of both psychoactive effects and unwanted side effects restricted to different enantiomers might be useful to understand strategy of synthesis. In this context, mostly racemic mixtures are produced, however, there are few exceptions. In-house analysis of real-life samples seized by Austrian police revealed that pure enantiomers of certain products are a result of educts as pure enantiomers, as it is the case for the production of crystal meth from ephedrine available as over-the-counter tablets [16]. These findings also correlate with a recent publication [17].

Enantioseparation of chiral NPS for analytic purposes are mainly performed via HPLC by means of various chiral columns, among them cellulose derivatives or cyclodextrins [18] as chiral selectors. Further successful work was done in this field by means of gas chromatography (GC). A comprehensive overview of reported methods for enantioseparation of NPS is given in a survey article [18]. Capillary electrophoresis (CE) counts as a complementary technique to HPLC and represents a powerful alternative for separation of enantiomers by means of simple method development and low sample and electrolyte consumption combined with inexpensive UV detection. In this field, previous work has already shown the potential of cyclodextrins as chiral additives in CE [19-21]. This chiral selector class comprises $6(\alpha-), 7(\beta-)$ or 8 $(\gamma$-cyclodextrin) cyclic glycopyranose units forming a truncated cone. Chiral recognition takes place by host-guest interaction via inclusion complexation as well as by additional hydrogen bondings or dipol-dipol interactions. Each enantiomer is intended to form a differently migrating complex with the chiral selector leading to two separated peaks in the electropherogram. Also, native cyclodextrins can undergo derivatisation of the hydroxy groups in position 2, 3 and 6 resulting in derivatives, which are determined by their degree of substitution (DS) and varying cone depths. Moreover, they possess low UV-absorption preventing detection problems. This makes cyclodextrins to the most often applied chiral selectors for CE [22].

First, cyclodextrin-assisted chiral separations of the parent compounds amphetamine and its derivatives as well as cathinone and methcathinone were reported by Lurie et al. already in 1994 [23]. Later, further amphetamine derivatives were separated using $\beta$-cyclodextrins but also sulfated $\gamma$-cyclodextrins [24-35]. In the sequel, successful enantioseparation of various cathinones [36-39] and benzofurans [40] as new compound classes of NPS was reported. Chiral separation of the parent compound ketamine was performed first in 1992 [41].

The present work ties in with the work of Hägele et al. [42,43], presenting also successful 
results of latest NPS involving further chiral substance classes by means of the non-charged native, acetyl- and 2-hydroxypropyl- $\beta-\mathrm{CD}$ as well as the negatively charged carboxymethyl- $\beta$ CD (DS 0.5). The aim is to extend the number of chiral separations of NPS. In total, 136 NPS were tested by carboxymethyl- $\beta$-CD (DS 3.5), carboxyethyl- $\beta-\mathrm{CD}$ and succinyl- $\beta$-CD. Because of their novelty, these NPS are partially not yet commercially available and were purchased from various internet shops or seized by Austrian police.

\section{Materials and Methods}

\subsection{Chemicals}

Carboxymethyl-, succinyl- and carboxyethyl- $\beta$-CD (degree of substitution 3.5 each) were bought from CycloLab Ltd. (Budapest, Hungary). Di-sodium hydrogen phosphate and diluted phosphoric acid were from Merck KGaA (Darmstadt, Germany) and water from Fisher Scientific (Loughborough, UK). All components were of analytical grade.

\subsection{Samples}

Analytes already commercially available were purchased from LGC Standards GmbH (Wesel, Germany) or Lipomed AG (Arlesheim, Switzerland). The majority of NPS were either bought from different online shops or represented real-life samples seized by Austrian police and provided for research purposes. Prior to experiments, their identity was confirmed by GC-MS Chemical structures and names of tested NPS are listed in Table 1. They were mainly available as hydrochloric acid salts.

Table 1.1 Structures and names of investigated NPS

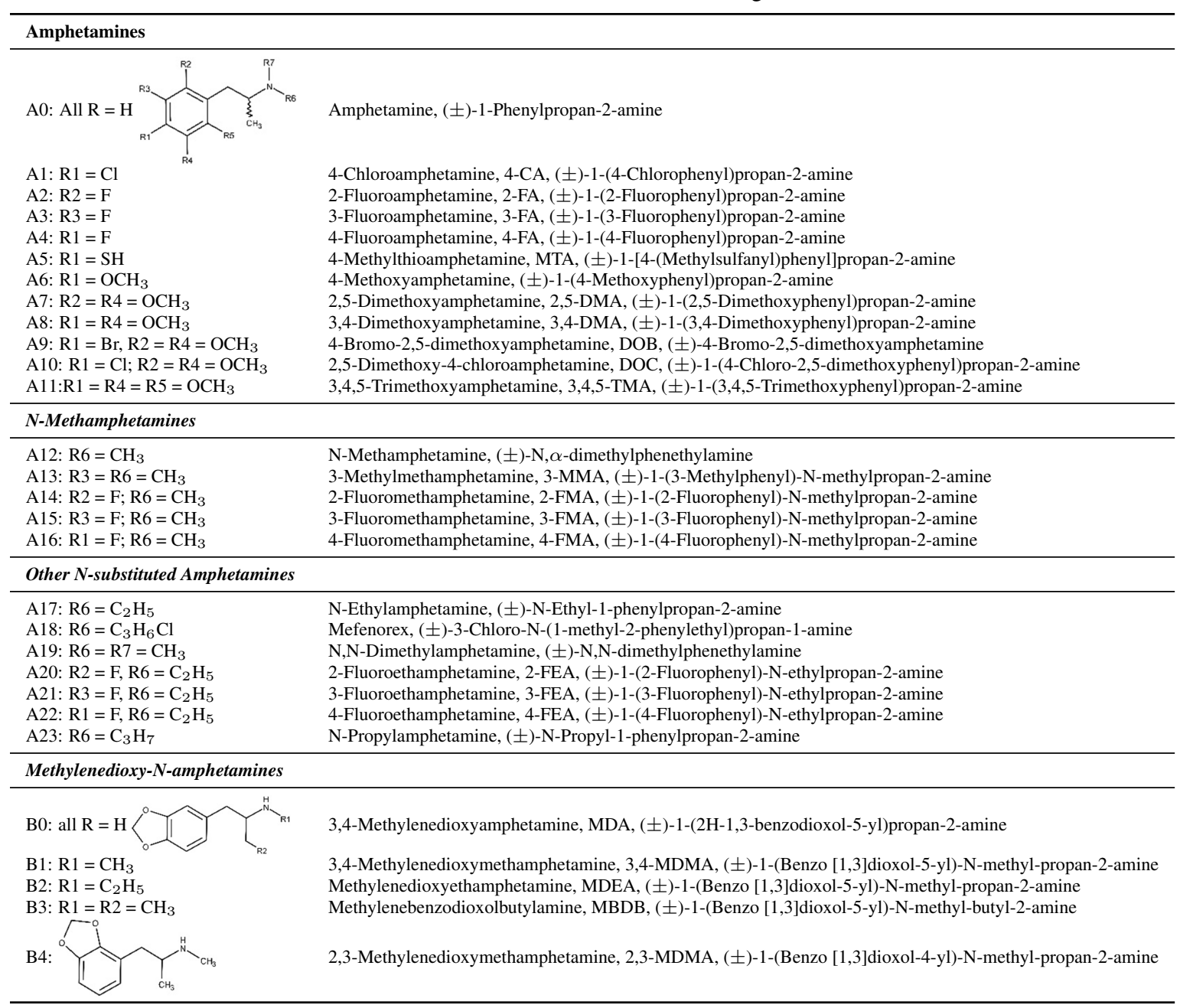


Table 1.2 Structures and names of investigated NPS

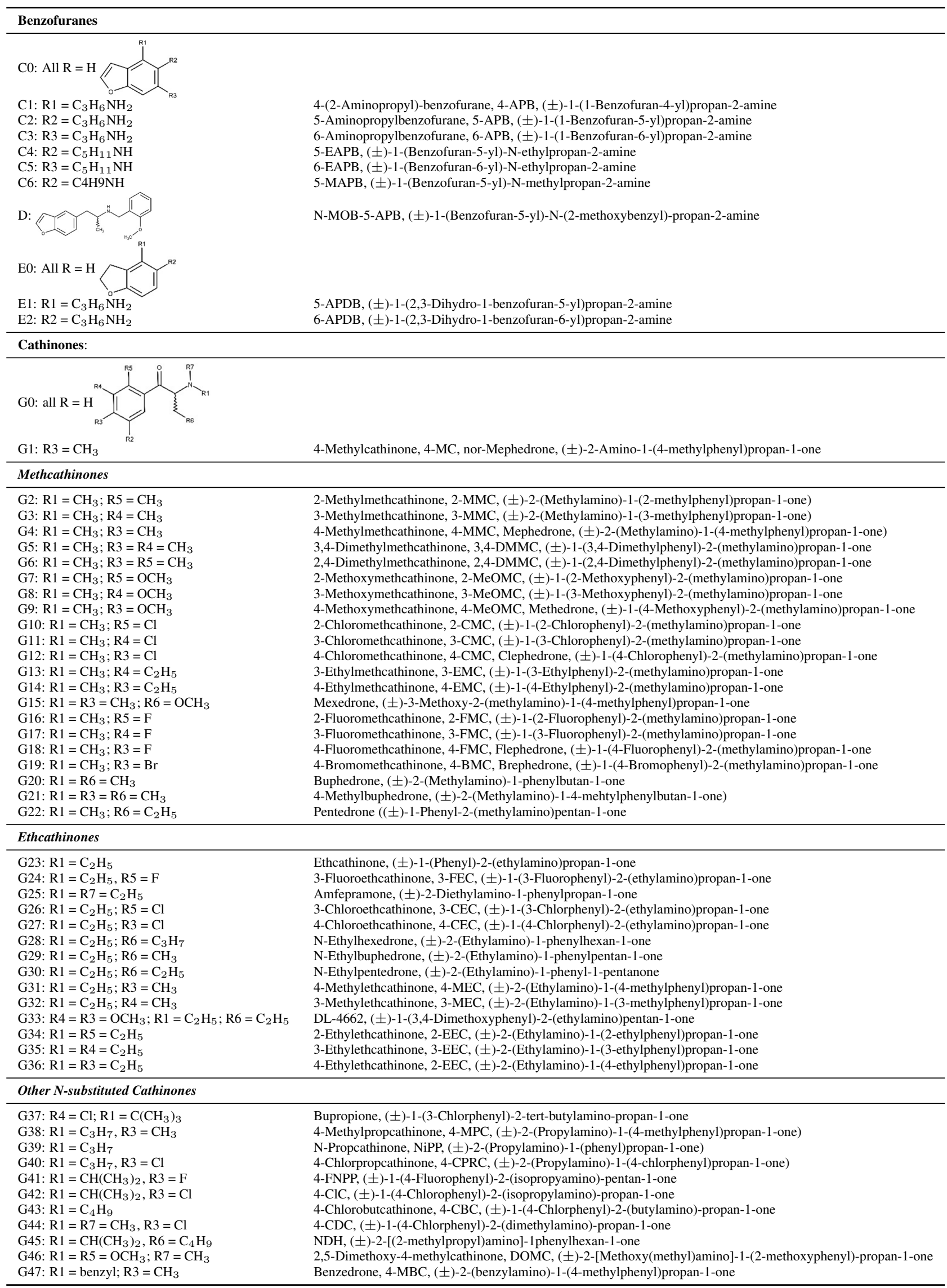


Table 1.3 Structures and names of investigated NPS

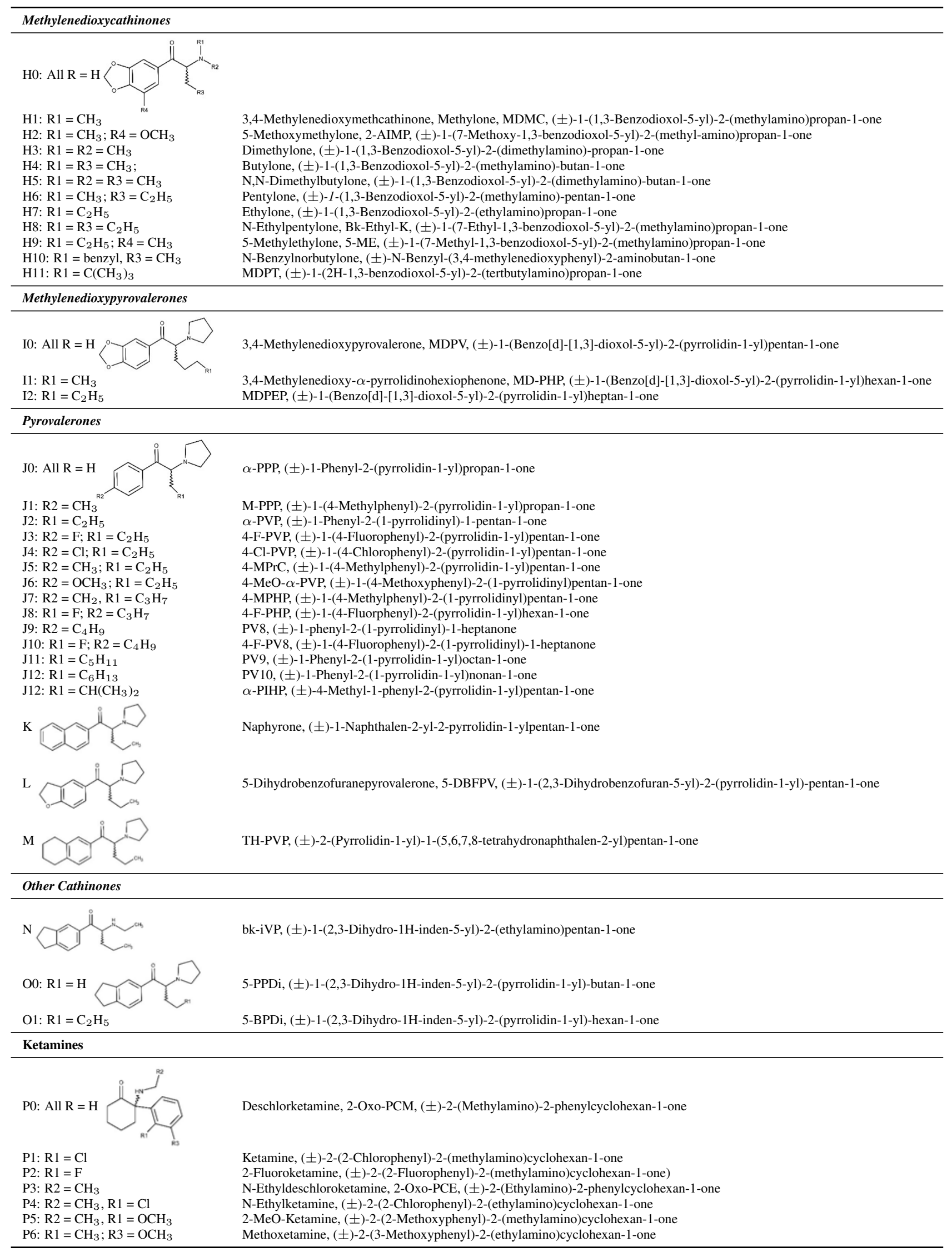


Table 1.4 Structures and names of investigated NPS

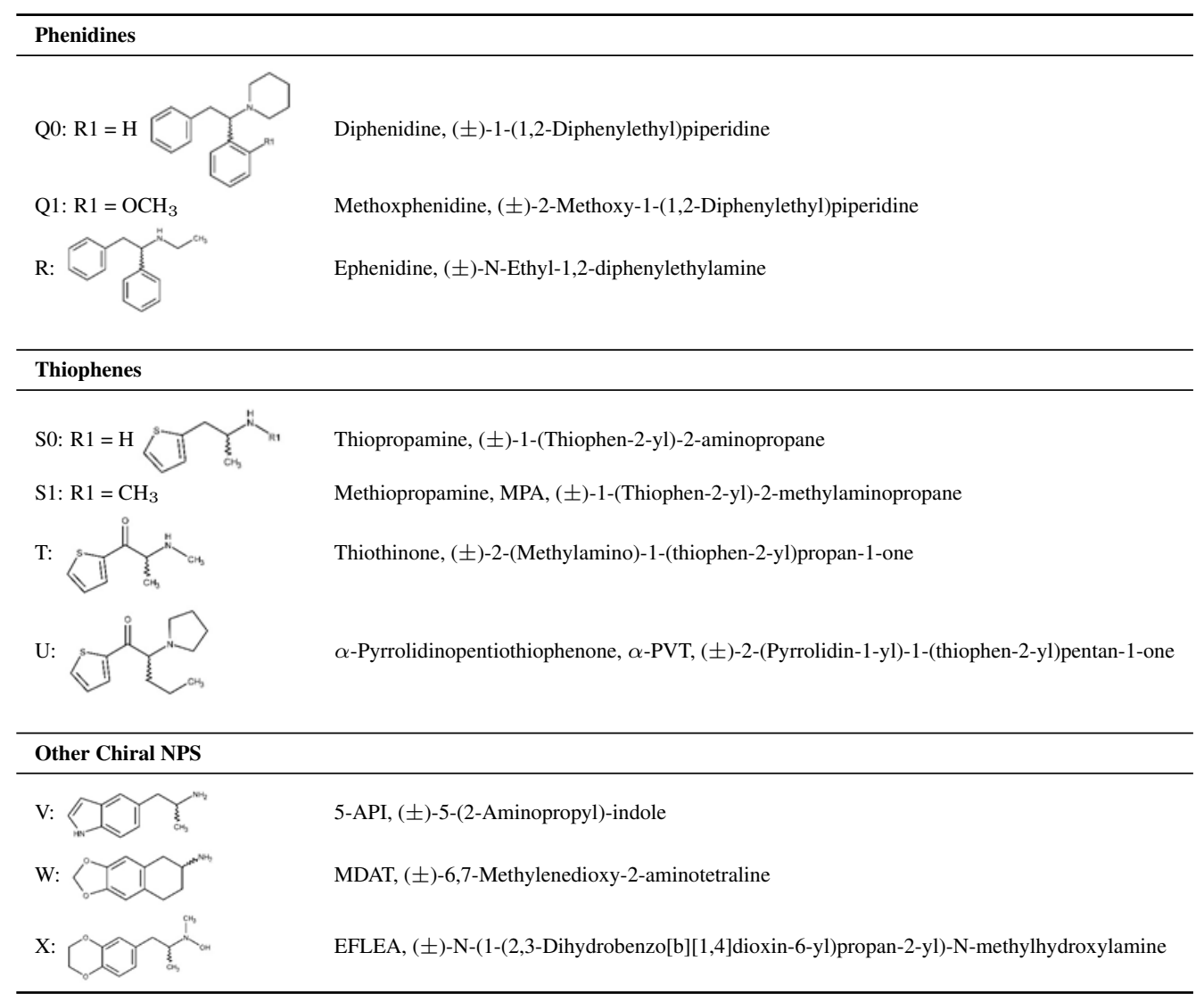

\subsection{Preparation of samples and background electrolyte}

Samples were dissolved in water $(1 \mathrm{mg} / \mathrm{ml})$ using an ultrasonic bath. Background electrolyte (BGE) consisted of aqueous $10 \mathrm{mM}$ di-sodium hydrogen phosphate and $10 \mathrm{mM}$ of the respective cyclodextrin. $\mathrm{pH}$ was adjusted to 2.5 with diluted phosphoric acid. Both, samples and BGE were filtered through a $0.45 \mu \mathrm{m}$ single-use syringe filter (Carl Roth, Karlsruhe, Germany).

\subsection{Instrumentation}

Measurements were carried out on a DAD equipped Agilent 7100 Capillary Electrophoresis using UV-detection at $209 \mathrm{~nm}$. The used voltage to cathode was set to $22 \mathrm{kV}$ for the BGE containing carboxymethyl- and carboxyethyl- $\beta-\mathrm{CD}$ and $26 \mathrm{kV}$ for the experiments with succinyl$\beta$-CD. Measurements using the succinyl- $\beta$-CD electrolyte were set to an autozero after 3 minutes due to absorption interference probably from succinate. Cassette temperature was set to $25^{\circ} \mathrm{C}$. Measurements were performed in fused silica capillaries (ID $50 \mu \mathrm{m}$ ) purchased from MircoQuartz (Munich, Germany) with total length of $68.5 \mathrm{~cm}$ and effective length of $60.0 \mathrm{~cm}$. Sample injection was conducted dynamically by pressure set to $10 \mathrm{mbar}$ for $5 \mathrm{~s}$ at the inlet vial.

\section{Results and Discussion}

\subsection{Comparison of cyclodextrins}

Overall, all three negatively charged $\beta$-cyclodextrins showed excellent separation ability for all tested substances groups. Rs values and migration times were comparable to the results of CM- $\beta$-CD (DS 0.5) in previous work. CM-, CE- and succinyl- $\beta$-CD represent negatively charged cyclodextrin derivatives. Apparently, the positively charged samples tend to show better interaction with these types of cyclodextrins thus leading to higher resolution. In addition, measurements using buffers containing negatively charged cyclodextrins were performed with lower voltage to secure an acceptable amount of Joule heat, however, resulting in longer migrations times. Migration times ranged from 4.7 to $17.6 \mathrm{~min}$ with native, acetyl- or 
hydroxypropyl- $\beta$-cyclodextrin. The use of negatively charged cyclodextrins produced migration times from 7.8 to $24.4 \mathrm{~min}$ with a few exceptions up to $47 \mathrm{~min}$. Table 2 provides migration times and Rs values for each analyte investigated.

In Table 3 the total number of successful enantioseparations by means of each cyclodextrin is compared. Table 3 combines the data from Hägele et al. $[42,43]$ with new measurements of the present work. Negatively charged cyclodextrins are shown to be superior particularly in terms of baseline separation (RS value $>2$ ). Mostly baseline separations were obtained with carboxymethyl- $\beta$-cyclodextrin. The comparison of the two carboxymethyl- $\beta$-cyclodextrins with different DS revealed differences in their enantioselectivity.

Table 2.1 Results of 136 investigated NPS

\begin{tabular}{|c|c|c|c|c|c|}
\hline & $\mathrm{t} 1$ (min) & $\mathrm{t} 2(\mathrm{~min})$ & $\alpha(\mathrm{t} 2 / \mathrm{t} 1)$ & Rs & Selector \\
\hline \multicolumn{6}{|l|}{ Amphetamines } \\
\hline & 10.296 & 10.481 & 1.0180 & 1.0 & Succinyl \\
\hline \multirow{2}{*}{ Amphetamine sulfate } & 12.694 & 12.884 & 1.0150 & 1.0 & \\
\hline & 16.043 & 16.457 & 1.0258 & 1.5 & CM 3.5 \\
\hline \multirow{3}{*}{ 4-Chloramphetamine } & 17.086 & 17.386 & 1.0176 & 1.2 & Succinyl \\
\hline & 22.740 & 23.172 & 1.0190 & 1.2 & $\mathrm{CE}$ \\
\hline & 23.060 & 23.512 & 1.0196 & 1.4 & CM 3.5 \\
\hline \multirow{3}{*}{ 2-F-Amphetamine } & 12.803 & 13.071 & 1.0209 & 1.0 & Succinyl \\
\hline & 15.727 & 16.088 & 1.0230 & 1.2 & $\mathrm{CE}$ \\
\hline & 17.316 & 17.999 & 1.0394 & 2.0 & CM 3.5 \\
\hline \multirow{3}{*}{ 3-F-Amphetamine } & 13.309 & 13.523 & 1.0161 & 1.0 & Succinyl \\
\hline & 15.934 & 16.210 & 1.0173 & 1.1 & $\mathrm{CE}$ \\
\hline & 17.178 & 17.531 & 1.0205 & 1.4 & CM 3.5 \\
\hline \multirow{3}{*}{ 4-F-Amphetamine } & 11.872 & 12.085 & 1.0179 & 1.1 & Succinyl \\
\hline & 15.590 & 15.812 & 1.0142 & 1.1 & \\
\hline & 15.140 & 15.378 & 1.0157 & 1.0 & CM 3.5 \\
\hline \multirow{3}{*}{ MTA (4-Methylthioamphetamine) } & 13.112 & n.d. & - & - & Succinyl \\
\hline & 19.564 & n.d. & - & - & $\mathrm{CE}$ \\
\hline & 28.472 & 28.681 & 1.0073 & 0.5 & CM 3.5 \\
\hline \multirow{3}{*}{ DOB (4-Br-2,5-DiMeO-Amphetamine) } & 9.462 & n.d. & - & - & Succinyl \\
\hline & 12.619 & n.d. & - & - & $\mathrm{CE}$ \\
\hline & 15.366 & n.d. & - & - & CM 3.5 \\
\hline \multirow{3}{*}{ DOC (2,4-DiMeO-4-Cl-Amphetamine) } & 12.510 & n.d. & - & - & Succinyl \\
\hline & 15.510 & n.d. & - & - & $\mathrm{CE}$ \\
\hline & 15.446 & n.d. & - & - & CM 3.5 \\
\hline \multirow{3}{*}{ 3,4-Dimethoxyamphetamine } & 8.622 & n.d. & - & - & Succinyl \\
\hline & 11.223 & n.d. & - & - & \\
\hline & 12.487 & 12.579 & 1.0074 & 0.1 & CM 3.5 \\
\hline \multirow{3}{*}{ 4-MeO-Amphetamine } & 11.448 & 11.637 & 1.0165 & 1.1 & Succinyl \\
\hline & 15.008 & 15.151 & 1.0095 & 0.7 & $\mathrm{CE}$ \\
\hline & 20.067 & 20.473 & 1.0202 & 1.3 & CM 3.5 \\
\hline \multirow{3}{*}{ 2,5-Dimethoxyamphetamine } & 9.305 & 9.404 & 1.0106 & 0.9 & Succinyl \\
\hline & 12.893 & 13.063 & 1.0132 & 1.0 & \\
\hline & 15.729 & 16.030 & 1.0191 & 1.4 & CM 3.5 \\
\hline \multirow{3}{*}{ 3,4,5-Trimethoxyamphetamine } & 8.771 & n.d. & - & - & Succinyl \\
\hline & 11.472 & n.d. & - & - & $\mathrm{CE}$ \\
\hline & 12.401 & n.d. & - & - & CM 3.5 \\
\hline \multicolumn{6}{|l|}{$N$-Methamphetamines } \\
\hline \multirow{3}{*}{ N-Methamphetamine } & 13.335 & 13.679 & 1.0258 & 1.5 & Succinyl \\
\hline & 13.480 & 13.758 & 1.0206 & 1.4 & \\
\hline & 16.581 & 17.004 & 1.0255 & 1.9 & CM 3.5 \\
\hline \multirow{3}{*}{ 2-FMA } & 12.295 & 12.611 & 1.0257 & 1.4 & Succinyl \\
\hline & 13.574 & 14.003 & 1.0316 & 1.9 & \\
\hline & 19.563 & 20.604 & 1.0532 & 4.4 & CM 3.5 \\
\hline \multirow{3}{*}{ 3-FMA } & 13.599 & 13.903 & 1.0224 & 1.3 & Succinyl \\
\hline & 17.525 & 17.900 & 1.0214 & 1.4 & \\
\hline & 17.544 & 17.918 & 1.0213 & 1.6 & CM 3.5 \\
\hline & 11.054 & 11.237 & 1.0166 & 0.9 & Succinyl \\
\hline 4-FMA & 12.477 & 12.640 & 1.0131 & 0.9 & \\
\hline & 17.600 & 17.898 & 1.0169 & 1.2 & CM 3.5 \\
\hline & 14.078 & 14.513 & 1.0309 & 2.2 & Succinyl \\
\hline 3-MMA & 18.096 & 18.698 & 1.0333 & 2.6 & \\
\hline & 18.078 & 18.680 & 1.0333 & 2.5 & CM 3.5 \\
\hline Cathinones & & & & & \\
\hline 4-Methylcathinone (4-MC) & 11.293 & n.d. & - & - & Succinyl \\
\hline & 13.891 & n.d. & - & - & $\mathrm{CE}$ \\
\hline & 16.170 & 16.352 & 1.0113 & 0.9 & CM 3.5 \\
\hline
\end{tabular}


Table 2.2 Results of 136 investigated NPS

\begin{tabular}{|c|c|c|c|c|c|}
\hline & $\mathrm{t} 1$ (min) & $\mathrm{t} 2(\mathrm{~min})$ & $\alpha(\mathrm{t} 2 / \mathrm{t} 1)$ & Rs & Selector \\
\hline \multicolumn{6}{|c|}{ Methylendioxy-/Methylendioxy-N-Amphetamines } \\
\hline & 14.991 & 15.152 & 1.0107 & 0.8 & \multirow{3}{*}{$\begin{array}{l}\text { Succinyl } \\
\text { CE } \\
\text { CM } 3.5\end{array}$} \\
\hline \multirow{2}{*}{ 3,4-MDA (3,4-Methylendioxyamph.) } & 16.098 & 16.359 & 1.0162 & 0.9 & \\
\hline & 25.924 & 26.299 & 1.0145 & 1.0 & \\
\hline \multirow{4}{*}{ 3,4-MDMA } & 13.217 & 13.476 & 1.0196 & 1.2 & \multirow{3}{*}{$\begin{array}{l}\text { Succinyl } \\
\text { CE } \\
\text { CM } 3.5\end{array}$} \\
\hline & 16.825 & 17.156 & 1.0197 & 1.1 & \\
\hline & 24.045 & 24.512 & 1.0194 & 1.5 & \\
\hline & 9.260 & 9.363 & 1.0111 & 1.0 & \multirow{3}{*}{$\begin{array}{l}\text { Succinyl } \\
\text { CE } \\
\text { CM } 3.5\end{array}$} \\
\hline \multirow{3}{*}{ 2,3-MDMA } & 10.932 & 11.036 & 1.0095 & 0.9 & \\
\hline & 12.466 & 12.647 & 1.0145 & 1.4 & \\
\hline & 16.710 & 17.072 & 1.0217 & 1.1 & \multirow{3}{*}{$\begin{array}{l}\text { Succinyl } \\
\text { CE } \\
\text { CM } 3.5\end{array}$} \\
\hline \multirow[t]{3}{*}{ MDEA } & 18.705 & 19.016 & 1.0166 & 1.0 & \\
\hline & 26.239 & 26.613 & 1.0143 & 1.0 & \\
\hline & 17.226 & 17.346 & 1.0070 & 0.7 & \multirow{3}{*}{$\begin{array}{l}\text { Succinyl } \\
\text { CE } \\
\text { CM } 3.5\end{array}$} \\
\hline \multirow[t]{2}{*}{ MBDB } & 18.943 & n.d. & - & - & \\
\hline & 26.188 & n.d. & - & - & \\
\hline \multicolumn{6}{|l|}{ Other N-substituted Amphetamines } \\
\hline \multirow{4}{*}{ N-Ethylamphetamine } & 13.404 & 13.786 & 1.0285 & 1.4 & Succinyl \\
\hline & 14.715 & 14.987 & 1.0185 & 1.0 & \\
\hline & 18.518 & 19.011 & 1.0266 & 1.7 & CM 3.5 \\
\hline & 14.462 & 14.793 & 1.0229 & 1.3 & Succinyl \\
\hline Mefenorex & 15.717 & 15.987 & 1.0172 & 1.2 & $\mathrm{CE}$ \\
\hline & 20.266 & 20.753 & 1.0240 & 1.8 & CM 3.5 \\
\hline & 11.695 & 12.088 & 1.0336 & 2.5 & Succinyl \\
\hline DMA (N,N,-Dimethylamphetamine) & 13.699 & 14.087 & 1.0283 & 1.9 & \\
\hline & 16.875 & 17.423 & 1.0325 & 2.0 & CM 3.5 \\
\hline & 13.811 & 14.203 & 1.0284 & 1.5 & Succinyl \\
\hline 2-F-Ethamphetamine & 15.074 & 15.525 & 1.0299 & 1.9 & $\mathrm{CE}$ \\
\hline & 18.531 & 19.340 & 1.0437 & 3.0 & CM 3.5 \\
\hline & 14.109 & 14.449 & 1.0241 & 1.4 & Succinyl \\
\hline 3-F-Ethamphetamine & 14.612 & 14.880 & 1.0183 & 1.1 & \\
\hline & 18.057 & 18.455 & 1.0220 & 1.8 & CM 3.5 \\
\hline & 12.443 & 12.730 & 1.0231 & 1.5 & Succinyl \\
\hline 4-F-Ethamphetamine & 13.750 & 13.928 & 1.0129 & 1.0 & $\mathrm{CE}$ \\
\hline & 17.907 & 18.180 & 1.0152 & 1.4 & CM 3.5 \\
\hline & 11.666 & 11.939 & 1.0234 & 1.4 & Succinyl \\
\hline N-Propylamphetamine & 15.032 & 15.484 & 1.0301 & 1.9 & \\
\hline & 18.387 & 19.188 & 1.0436 & 3.3 & CM 3.5 \\
\hline Benzofuranes & & & & & \\
\hline & 8.397 & 8.508 & 1.0132 & 1.2 & Succinyl \\
\hline 4-APB & 11.277 & 11.436 & 1.0141 & 0.9 & $\mathrm{CE}$ \\
\hline & 13.955 & 14.265 & 1.0222 & 1.6 & CM 3.5 \\
\hline & 14.355 & n.d. & - & - & Succinyl \\
\hline $5-\mathrm{APB}$ & 20.192 & 20.409 & 1.0107 & 1.0 & $\mathrm{CE}$ \\
\hline & 15.645 & 15.999 & 1.0226 & 2.9 & CM 3.5 \\
\hline & 10.850 & 11.036 & 1.0171 & 1.3 & Succinyl \\
\hline 5-APDB & 15.061 & 15.317 & 1.0170 & 1.3 & $\mathrm{CE}$ \\
\hline & 14.053 & 14.331 & 1.0198 & 2.7 & CM 3.5 \\
\hline & 16.930 & 17.296 & 1.0216 & 1.4 & Succinyl \\
\hline 5-EAPB & 19.377 & 19.658 & 1.0145 & 1.3 & $\mathrm{CE}$ \\
\hline & 28.557 & 29.105 & 1.0192 & 1.6 & CM 3.5 \\
\hline & 16.055 & 16.458 & 1.0251 & 1.7 & Succinyl \\
\hline 5-MAPB & 18.298 & 18.625 & 1.0179 & 1.6 & $\mathrm{CE}$ \\
\hline & 24.805 & 25.322 & 1.0208 & 2.5 & CM 3.5 \\
\hline & 14.366 & n.d. & - & - & Succinyl \\
\hline N-MOB-5-APB & 20.648 & n.d. & - & - & \\
\hline & 27.070 & n.d. & - & - & CM 3.5 \\
\hline & 14.812 & n.d. & - & - & Succinyl \\
\hline 6-APB & 20.985 & 21.217 & 1.0111 & 1.3 & $\mathrm{CE}$ \\
\hline & 15.014 & 15.333 & 1.0212 & 3.4 & CM 3.5 \\
\hline & 14.379 & n.d. & - & - & Succinyl \\
\hline 6-APDB & 18.773 & 19.022 & 1.0133 & 0.9 & $\mathrm{CE}$ \\
\hline & 28.518 & 29.051 & 1.0187 & 1.3 & CM 3.5 \\
\hline & 15.045 & n.d. & - & - & Succinyl \\
\hline 6-ЕАРB & 21.439 & 21.632 & 1.0090 & 0.7 & $\mathrm{CE}$ \\
\hline & 30.787 & n.d. & - & - & CM 3.5 \\
\hline
\end{tabular}

Note: Results of chiral separation attempts using succinyl-, carboxyethyl-, and carboxymethyl- $\beta$-CD, degree of substitution each 3.5 , n.d. = not detected 
Table 2.3 Results of 136 investigated NPS

\begin{tabular}{|c|c|c|c|c|c|}
\hline & $\mathrm{t} 1(\min )$ & $\mathrm{t} 2(\mathrm{~min})$ & $\alpha(\mathrm{t} 2 / \mathrm{t} 1)$ & Rs & Selector \\
\hline \multicolumn{6}{|l|}{ Methcathinones } \\
\hline \multirow{3}{*}{ 2-MMC } & 9.244 & n.d. & - & - & Succinyl \\
\hline & 11.588 & n.d. & - & - & $\mathrm{CE}$ \\
\hline & 13.965 & n.d. & - & - & CM 3.5 \\
\hline \multirow{3}{*}{ 3-MMC } & 10.890 & 11.073 & 1.0168 & 1.1 & Succinyl \\
\hline & 13.204 & 13.464 & 1.0197 & 1.2 & \\
\hline & 17.440 & 17.884 & 1.0255 & 2.1 & CM 3.5 \\
\hline \multirow{3}{*}{ Mephedrone (4-MMC) } & 11.389 & n.d. & - & - & Succinyl \\
\hline & 14.239 & 14.348 & 1.0077 & 0.6 & $\mathrm{CE}$ \\
\hline & 20.811 & 21.234 & 1.0203 & 1.4 & CM 3.5 \\
\hline \multirow{3}{*}{ 3,4-DMMC } & 13.459 & 13.747 & 1.0214 & 1.2 & Succinyl \\
\hline & 16.649 & 16.958 & 1.0186 & 1.1 & \\
\hline & 23.395 & 24.080 & 1.0293 & 2.0 & CM 3.5 \\
\hline \multirow{3}{*}{ 2,4-DMMC } & 8.735 & n.d. & - & - & Succinyl \\
\hline & 12.852 & n.d. & - & - & $\mathrm{CE}$ \\
\hline & 14.390 & 14.585 & 0.2815 & 1.4 & CM 3.5 \\
\hline \multirow{3}{*}{ 3-MeO-MC } & 12.671 & 12.799 & 1.0101 & 0.7 & Succinyl \\
\hline & 14.688 & 15.104 & 1.0283 & 2.1 & $\mathrm{CE}$ \\
\hline & 17.446 & 18.162 & 1.0410 & 3.5 & CM 3.5 \\
\hline \multirow{3}{*}{ 2-MeO-MC } & 8.272 & n.d. & - & - & Succinyl \\
\hline & 11.449 & 11.510 & 1.0053 & 0.5 & $\mathrm{CE}$ \\
\hline & 12.698 & 12.983 & 1.0224 & 1.8 & CM 3.5 \\
\hline \multirow{3}{*}{ Methedrone (4-MeO-MC) } & 10.291 & 10.414 & 1.0120 & 0.9 & Succinyl \\
\hline & 14.429 & n.d. & - & - & \\
\hline & 19.399 & n.d. & - & - & CM 3.5 \\
\hline \multirow{3}{*}{ 2-CMC } & 16.609 & n.d. & - & - & Succinyl \\
\hline & n.d. & n.d. & - & - & $\mathrm{CE}$ \\
\hline & 28.025 & n.d. & - & - & CM 3.5 \\
\hline \multirow{3}{*}{ 3-CMC } & 12.078 & 12.303 & 1.0186 & 1.6 & Succinyl \\
\hline & 14.540 & 14.685 & 1.0100 & 0.7 & \\
\hline & 18.848 & 19.016 & 1.0089 & 0.6 & CM 3.5 \\
\hline \multirow{3}{*}{ 4-CMC } & 11.978 & 12.166 & 1.0157 & 1.1 & Succinyl \\
\hline & 14.814 & n.d. & - & - & $\mathrm{CE}$ \\
\hline & 18.938 & 19.114 & 1.0093 & 0.7 & CM 3.5 \\
\hline \multirow{3}{*}{ 3-EMC } & 11.171 & 11.499 & 1.0294 & 2.0 & Succinyl \\
\hline & 16.516 & 16.745 & 1.0139 & 1.0 & \\
\hline & 21.026 & 21.346 & 1.0152 & 1.1 & CM 3.5 \\
\hline & 14.035 & 14.197 & 1.0115 & 0.9 & Succinyl \\
\hline 4-EMC & 17.762 & 17.898 & 1.0077 & 0.6 & $\mathrm{CE}$ \\
\hline & 24.169 & 24.626 & 1.0189 & 1.6 & CM 3.5 \\
\hline & 11.072 & 11.217 & 1.0131 & 0.8 & Succinyl \\
\hline Mexedrone & 14.029 & 14.105 & 1.0054 & 0.6 & \\
\hline & 19.399 & n.d. & - & - & CM 3.5 \\
\hline & 17.769 & n.d. & - & - & CM 3.5 \\
\hline & 7.877 & n.d. & - & - & Succinyl \\
\hline 2-FMC & 12.187 & 12.413 & 1.0185 & 2.8 & $\mathrm{CE}$ \\
\hline & 15.033 & 15.637 & 1.0402 & 4.0 & CM 3.5 \\
\hline & 9.452 & n.d. & - & - & Succinyl \\
\hline 3-FMC & 11.552 & 11.607 & 1.0048 & 0.5 & $\mathrm{CE}$ \\
\hline & 14.192 & 14.372 & 1.0127 & 1.2 & CM 3.5 \\
\hline & 9.037 & 9.120 & 1.0092 & 0.7 & Succinyl \\
\hline 4-FMC & 11.251 & n.d. & - & - & $\mathrm{CE}$ \\
\hline & 13.568 & n.d. & - & - & CM 3.5 \\
\hline & 13.417 & 13.604 & 1.0139 & 0.9 & Succinyl \\
\hline 4-BMC & 16.913 & n.d. & - & - & $\mathrm{CE}$ \\
\hline & 22.520 & 22.717 & 1.0087 & 0.8 & CM 3.5 \\
\hline & 10.913 & 11.359 & 1.0409 & 2.3 & Succinyl \\
\hline Buphedrone & 13.623 & n.d. & - & - & $\mathrm{CE}$ \\
\hline & 16.275 & n.d. & - & - & CM 3.5 \\
\hline & 13.115 & 13.721 & 1.0462 & 2.9 & Succinyl \\
\hline 4-Methylbuphedrone & 16.493 & n.d. & - & - & $\mathrm{CE}$ \\
\hline & 21.165 & n.d. & - & - & CM 3.5 \\
\hline & 11.644 & 11.837 & 1.0166 & 1.1 & Succinyl \\
\hline Pentedrone & 13.902 & 14.413 & 1.0368 & 2.8 & $\mathrm{CE}$ \\
\hline & 16.552 & 17.319 & 1.0463 & 3.9 & CM 3.5 \\
\hline
\end{tabular}

Note: Results of chiral separation attempts using succinyl-, carboxyethyl-, and carboxymethyl- $\beta$-CD, degree of substitution each 3.5 , n.d. $=$ not detected 
Table 2.4 Results of 136 investigated NPS

\begin{tabular}{|c|c|c|c|c|c|}
\hline & $\mathrm{t} 1(\min )$ & $\mathrm{t} 2(\mathrm{~min})$ & $\alpha(\mathrm{t} 2 / \mathrm{t} 1)$ & Rs & Selector \\
\hline \multicolumn{6}{|l|}{ Ethcathinones } \\
\hline \multirow{4}{*}{ Ethcathinone } & 10.200 & 10.274 & 1.0073 & 0.6 & Succinyl \\
\hline & 11.137 & 11.237 & 1.0090 & 0.7 & \\
\hline & 14.184 & 14.403 & 1.0154 & 1.2 & CM 3.5 \\
\hline & 12.153 & 12.326 & 1.0142 & 1.2 & Succinyl \\
\hline \multirow[t]{3}{*}{ Amphepramone } & 13.434 & 13.827 & 1.0293 & 2.2 & $\mathrm{CE}$ \\
\hline & 17.860 & 18.592 & 1.0410 & 3.6 & CM 3.5 \\
\hline & 12.236 & 12.491 & 1.0208 & 1.3 & Succinyl \\
\hline \multirow{3}{*}{ 3-CEC } & 13.886 & 14.008 & 1.0088 & 0.6 & $\mathrm{CE}$ \\
\hline & 21.697 & 21.908 & 1.0097 & 0.6 & CM 3.5 \\
\hline & 12.291 & 12.539 & 1.0202 & 1.3 & Succinyl \\
\hline \multirow[t]{3}{*}{ 4-CEC } & 13.733 & n.d. & - & - & $\mathrm{CE}$ \\
\hline & 21.827 & 21.927 & 1.0046 & 0.4 & CM 3.5 \\
\hline & 10.776 & 10.957 & 1.0168 & 1.5 & Succinyl \\
\hline \multirow{3}{*}{ DL-4662 } & 12.294 & 12.355 & 1.0050 & 0.5 & $\mathrm{CE}$ \\
\hline & 14.982 & n.d. & - & - & CM 3.5 \\
\hline & 12.130 & 12.488 & 1.0295 & 2.0 & Succinyl \\
\hline \multirow[t]{3}{*}{ N-Ethylhexedrone } & 14.066 & 14.407 & 1.0242 & 1.7 & $\mathrm{CE}$ \\
\hline & 18.317 & 18.951 & 1.0346 & 2.9 & CM 3.5 \\
\hline & 10.952 & 11.100 & 1.0135 & 0.8 & Succinyl \\
\hline 3-MEC & 12.719 & 12.909 & 1.0149 & 1.2 & $\mathrm{CE}$ \\
\hline & 16.035 & 16.321 & 1.0178 & 1.4 & CM 3.5 \\
\hline & 11.912 & n.d. & - & - & Succinyl \\
\hline 4-MEC & 13.611 & 13.706 & 1.0070 & 0.6 & $\mathrm{CE}$ \\
\hline & 17.803 & 18.071 & 1.0151 & 1.2 & CM 3.5 \\
\hline & 11.703 & 12.143 & 1.0376 & 2.7 & Succinyl \\
\hline $\mathrm{N}$-Ethylbuphedrone & 13.692 & n.d. & - & - & CE \\
\hline & 16.887 & n.d. & - & - & CM 3.5 \\
\hline & 10.539 & 10.711 & 1.0163 & 1.3 & Succinyl \\
\hline N-Ethylpentedrone & 12.374 & 12.439 & 1.0053 & 0.6 & $\mathrm{CE}$ \\
\hline & 14.651 & n.d. & - & - & CM 3.5 \\
\hline & 9.698 & 9.748 & 1.0052 & 0.6 & Succinyl \\
\hline 2-Ethylethcathinone & 12.266 & n.d. & - & - & $\mathrm{CE}$ \\
\hline & 15.399 & n.d. & - & - & CM 3.5 \\
\hline & 12.707 & 12.966 & 1.0204 & 1.7 & Succinyl \\
\hline 3-Ethylethcathinone & 15.630 & 15.734 & 1.0067 & 0.6 & $\mathrm{CE}$ \\
\hline & 21.661 & 21.834 & 1.0080 & 0.9 & CM 3.5 \\
\hline & 12.067 & 12.299 & 1.0192 & 1.5 & Succinyl \\
\hline 4-Ethylethcathinone & 14.238 & n.d. & - & - & $\mathrm{CE}$ \\
\hline & 17.724 & n.d. & - & - & CM 3.5 \\
\hline & 12.240 & 12.349 & 1.0089 & 0.8 & Succinyl \\
\hline 3-F-Ethcathinone & 15.208 & 15.379 & 1.0112 & 0.9 & $\mathrm{CE}$ \\
\hline & 14.993 & 15.162 & 1.0113 & 0.9 & CM 3.5 \\
\hline Other $N$-substituted Cath & & & & & \\
\hline 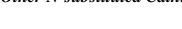 & 14.159 & 15.226 & 1.0754 & 4.7 & Succinyl \\
\hline Bupropione & 15.902 & 16.227 & 1.0204 & 1.3 & $\mathrm{CE}$ \\
\hline & 22.277 & 22.896 & 1.0278 & 1.6 & CM 3.5 \\
\hline & 13.952 & 14.316 & 1.0261 & 1.6 & Succinyl \\
\hline 4-MPC & 16.505 & n.d. & - & - & $\mathrm{CE}$ \\
\hline & 23.394 & n.d. & - & - & CM 3.5 \\
\hline & 12.511 & n.d. & - & - & Succinyl \\
\hline N-Propcathinone (NiPP) & 15.152 & 15.454 & 1.0199 & 1.4 & $\mathrm{CE}$ \\
\hline & 19.305 & 19.790 & 1.0251 & 1.8 & CM 3.5 \\
\hline & 14.379 & 14.760 & 1.0265 & 2.0 & Succinyl \\
\hline 4-CPRC & 17.036 & 17.393 & 1.0210 & 1.6 & $\mathrm{CE}$ \\
\hline & 23.014 & n.d. & - & - & CM 3.5 \\
\hline & 11.559 & 11.674 & 1.0099 & 0.8 & Succinyl \\
\hline 4-FNPP & 14.019 & 14.425 & 1.0290 & 2.2 & $\mathrm{CE}$ \\
\hline & 16.736 & 16.833 & 1.0058 & 0.6 & CM 3.5 \\
\hline & 13.558 & 13.857 & 1.0221 & 1.6 & Succinyl \\
\hline 4-Cl-C & 15.766 & n.d. & - & - & $\mathrm{CE}$ \\
\hline t-C & 20.576 & n.d. & 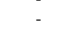 & 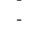 & CM 3.5 \\
\hline & 13.162 & 13.502 & 1.0258 & 1.4 & Succinyl \\
\hline 4-CBC & 15.081 & n.d. & - & - & $\mathrm{CE}$ \\
\hline & 18.966 & n.d. & - & - & CM 3.5 \\
\hline & 11.960 & 12.231 & 1.0227 & 1.5 & Succinyl \\
\hline 4-CDC & 13.478 & $\begin{array}{l}\text { n.d. } \\
\text { nes. }\end{array}$ & 1.022 & - & $\mathrm{CE}$ \\
\hline & 15.918 & n.d. & - & - & CM 3.5 \\
\hline & 12.972 & n.d. & - & - & Succinyl \\
\hline NDH & 14.825 & 15.226 & 1.0270 & 2.1 & $\mathrm{CE}$ \\
\hline & 18.205 & 18.868 & 1.0364 & 3.0 & CM 3.5 \\
\hline & 11.873 & n.d. & - & - & Succinyl \\
\hline DOMC & 16.274 & 16.380 & 1.0065 & 0.6 & $\mathrm{CE}$ \\
\hline & 20.141 & 20.896 & 1.0375 & 2.0 & CM 3.5 \\
\hline & 11.737 & n.d. & - & - & Succinyl \\
\hline 4-MBC (Benzedrone) & 14.943 & 15.070 & 1.0085 & 0.9 & $\mathrm{CE}$ \\
\hline & 17.715 & 17.984 & 1.0152 & 1.3 & CM 3.5 \\
\hline
\end{tabular}

Note: Results of chiral separation attempts using succinyl-, carboxyethyl-, and carboxymethyl- $\beta$-CD, degree of substitution each 3.5 , n.d. $=$ not detected 
Table 2.5 Results of 136 investigated NPS

\begin{tabular}{|c|c|c|c|c|c|}
\hline & $\mathrm{t} 1(\mathrm{~min})$ & t2 $(\min )$ & $\alpha(\mathrm{t} 2 / \mathrm{t} 1)$ & Rs & Selector \\
\hline \multicolumn{6}{|l|}{ Pyrovalerone } \\
\hline & 14.945 & 15.295 & 1.0234 & 1.5 & Succinyl \\
\hline \multirow[t]{2}{*}{ PV8 } & 16.704 & n.d. & - & - & $\mathrm{CE}$ \\
\hline & 26.789 & 27.106 & 1.0118 & 0.7 & CM 3.5 \\
\hline \multirow{3}{*}{ 4-F-PV8 } & 14.319 & 14.531 & 1.0148 & 1.3 & Succinyl \\
\hline & 15.750 & n.d. & - & - & $\mathrm{CE}$ \\
\hline & 20.938 & 21.33 & 1.0187 & 1.4 & CM 3.5 \\
\hline \multirow{3}{*}{$\alpha-\mathrm{PVP}$} & 13.554 & 13.976 & 1.0311 & 2.1 & Succinyl \\
\hline & 14.561 & 15.140 & 1.0398 & 2.8 & $\mathrm{CE}$ \\
\hline & 23.086 & 24.614 & 1.0662 & 4.3 & CM 3.5 \\
\hline \multirow{3}{*}{ 4-Cl-PVP } & 15.807 & 16.797 & 1.0626 & 4.3 & Succinyl \\
\hline & 17.039 & 17.406 & 1.0215 & 1.6 & \\
\hline & 24438 & n.d. & - & - & CM 3.5 \\
\hline \multirow{3}{*}{ 4F-PVP } & 16.198 & 16.571 & 1.0230 & 1.7 & Succinyl \\
\hline & 18.856 & n.d. & - & - & $\mathrm{CE}$ \\
\hline & 26.792 & 27.008 & 1.0081 & 0.7 & CM 3.5 \\
\hline \multirow{3}{*}{ 4-MeO- $\alpha$-PVP } & 15.423 & 15.906 & 1.0313 & 2.5 & Succinyl \\
\hline & 17.046 & 17.570 & 1.0307 & 2.4 & \\
\hline & 28.181 & 29.391 & 1.0429 & 3.2 & CM 3.5 \\
\hline \multirow{3}{*}{ 4-MPrC } & 18.032 & n.d. & - & - & Succinyl \\
\hline & 21.093 & n.d. & - & - & CE \\
\hline & 41.095 & 41.693 & 1.0146 & 1.0 & CM 3.5 \\
\hline \multirow{3}{*}{ PV9 } & 16.266 & 16.660 & 1.0242 & 1.6 & Succinyl \\
\hline & 18.699 & n.d. & - & - & \\
\hline & 32.237 & 32.379 & 1.0044 & 0.3 & CM 3.5 \\
\hline \multirow{3}{*}{$\alpha$-PPP } & 10.986 & 11.580 & 1.0541 & 3.2 & Succinyl \\
\hline & 11.946 & 12.215 & 1.0225 & 1.4 & $\mathrm{CE}$ \\
\hline & 15.933 & 16.372 & 1.0276 & 1.0 & CM 3.5 \\
\hline \multirow{3}{*}{ M-PPP } & 13.147 & 13.731 & 1.0444 & 2.7 & Succinyl \\
\hline & 14.199 & 14.448 & 1.0175 & 1.2 & \\
\hline & 20.317 & 20.813 & 1.0244 & 1.3 & CM 3.5 \\
\hline \multirow{3}{*}{$\alpha$-PIHP } & 15.513 & 15.786 & 1.0176 & 1.1 & Succinyl \\
\hline & 17.031 & 17.182 & 1.0089 & 0.6 & $\mathrm{CE}$ \\
\hline & 28.810 & 29.610 & 1.0278 & 1.7 & CM 3.5 \\
\hline \multirow{3}{*}{ 4F-PHP } & 12.641 & 12.833 & 1.0152 & 1.1 & Succinyl \\
\hline & 14.038 & 14.343 & 1.0217 & 1.6 & $\mathrm{CE}$ \\
\hline & 19.670 & 19.868 & 1.0101 & 0.9 & CM 3.5 \\
\hline \multirow{3}{*}{ Naphyrone } & 17.015 & 17.232 & 1.0128 & 0.9 & Succinyl \\
\hline & 18.530 & 18.834 & 1.0164 & 1.2 & $\mathrm{CE}$ \\
\hline & 31.496 & 32.304 & 1.0257 & 1.8 & CM 3.5 \\
\hline \multirow{3}{*}{ 4-MPHP } & 15.560 & 15.832 & 1.0175 & 1.3 & Succinyl \\
\hline & 17.152 & 17.306 & 1.0090 & 0.7 & $\mathrm{CE}$ \\
\hline & 23.193 & 23.574 & 1.0164 & 1.1 & CM 3.5 \\
\hline & 17.144 & 17.383 & 1.0139 & 1.0 & Succinyl \\
\hline PV10 & 20.596 & n.d. & - & - & $\mathrm{CE}$ \\
\hline & 29.053 & n.d. & - & - & CM 3.5 \\
\hline & 14.630 & 15.082 & 1.0309 & 2.6 & Succinyl \\
\hline 5-DBFPV & 21.930 & 22.207 & 1.0126 & 1.3 & $\mathrm{CE}$ \\
\hline & 24.211 & 24.625 & 1.0171 & 1.7 & CM 3.5 \\
\hline & 16.752 & n.d. & - & - & Succinyl \\
\hline TH-PVP & 23.491 & n.d. & - & - & $\mathrm{CE}$ \\
\hline & 28.836 & n.d. & - & - & CM 3.5 \\
\hline Methylenedixoyethcathinones & & & & & \\
\hline & 11.794 & n.d. & - & - & Succinyl \\
\hline Ethylone & 15.590 & 15.743 & 1.0098 & 0.9 & $\mathrm{CE}$ \\
\hline & 18.933 & 19.208 & 1.0145 & 1.2 & CM 3.5 \\
\hline & 14.716 & n.d. & - & - & Succinyl \\
\hline N-Ethylpentylone (Bk-Ethyl-K) & 18.553 & 18.974 & 1.0227 & 1.8 & $\mathrm{CE}$ \\
\hline & 24.010 & 24.807 & 1.0332 & 2.8 & CM 3.5 \\
\hline & 12.662 & n.d. & - & - & Succinyl \\
\hline 5-ME & 15.570 & 15.724 & 1.0099 & 1.0 & $\mathrm{CE}$ \\
\hline & 21.623 & 22.037 & 1.0191 & 1.3 & CM 3.5 \\
\hline
\end{tabular}

Note: Results of chiral separation attempts using succinyl-, carboxyethyl-, and carboxymethyl- $\beta$-CD, degree of substitution each 3.5 , n.d. = not detected 
Table 2.6 Results of 136 investigated NPS

\begin{tabular}{|c|c|c|c|c|c|}
\hline & $\mathrm{t} 1$ (min) & $\mathrm{t} 2(\min )$ & $\alpha(\mathrm{t} 2 / \mathrm{t} 1)$ & Rs & Selector \\
\hline \multicolumn{6}{|l|}{ Ketamines } \\
\hline & 10.036 & n.d. & - & - & Succinyl \\
\hline \multirow[t]{2}{*}{ Ketamine } & 14.288 & 14.369 & 1.0057 & 0.5 & $\mathrm{CE}$ \\
\hline & 15.700 & n.d. & - & - & CM 3.5 \\
\hline \multirow{3}{*}{ N-Ehtylketamine } & 10.736 & 10.890 & 1.0143 & 1.3 & Succinyl \\
\hline & 14.746 & n.d. & - & - & $\mathrm{CE}$ \\
\hline & 16.446 & n.d. & - & - & CM 3.5 \\
\hline \multirow{3}{*}{ Methoxetamine } & 11.220 & 11.665 & 1.0397 & 2.5 & Succinyl \\
\hline & 15.758 & 15.956 & 1.0126 & 1.0 & $\mathrm{CE}$ \\
\hline & 17.576 & 18.006 & 1.0245 & 1.3 & CM 3.5 \\
\hline \multirow{3}{*}{ 2-Oxо-PCE } & 10.076 & 10.378 & 1.0300 & 2.0 & Succinyl \\
\hline & 13.412 & 13.449 & 1.0028 & 0.3 & $\mathrm{CE}$ \\
\hline & 14.905 & 15.051 & 1.0098 & 0.9 & CM 3.5 \\
\hline \multirow{3}{*}{ 2-Охо-РСМ } & 9.877 & 10.351 & 1.0480 & 3.6 & Succinyl \\
\hline & 12.571 & 12.755 & 1.0146 & 1.1 & \\
\hline & 13.610 & 13.855 & 1.0180 & 1.4 & CM 3.5 \\
\hline \multirow{3}{*}{ 2-F-Ketamine } & 10.157 & 10.313 & 1.0154 & 1.3 & Succinyl \\
\hline & 12.993 & 13.038 & 1.0035 & 0.4 & $\mathrm{CE}$ \\
\hline & 15.672 & 15.767 & 1.0061 & 0.6 & CM 3.5 \\
\hline \multirow{3}{*}{ 2-MeO-Ketamine } & 11.278 & $11.97 ß$ & - & - & Succinyl \\
\hline & 15.751 & 15.966 & 1.0136 & 1.0 & \\
\hline & 17.296 & 17.709 & 1.0239 & 1.5 & CM 3.5 \\
\hline \multicolumn{6}{|l|}{ Phenidines } \\
\hline \multirow{3}{*}{ Diphenidine } & 16.457 & n.d. & - & - & Succinyl \\
\hline & 21.485 & n.d. & - & - & CE \\
\hline & 25.215 & n.d. & - & - & CM 3.5 \\
\hline \multirow{3}{*}{ Methoxyphenidine } & 16.643 & n.d. & - & - & Succinyl \\
\hline & 22.384 & n.d. & - & - & $\mathrm{CE}$ \\
\hline & 27.772 & n.d. & - & - & CM 3.5 \\
\hline \multirow{3}{*}{ Ephenidine } & 15.498 & 15.928 & 1.0277 & 2.0 & Succinyl \\
\hline & 20.465 & 20.669 & 1.0100 & 0.9 & \\
\hline & 30.075 & n.d. & - & - & CM 3.5 \\
\hline \multicolumn{6}{|l|}{ Thiophenes } \\
\hline \multirow{3}{*}{$\alpha$-PVT } & 12.931 & 13.175 & 1.0189 & 1.7 & Succinyl \\
\hline & 14.091 & 14.380 & 1.0205 & 1.4 & $\mathrm{CE}$ \\
\hline & 17.559 & 18.094 & 1.0305 & 2.6 & CM 3.5 \\
\hline \multirow{3}{*}{ Thiothinone } & 8.783 & n.d. & - & - & Succinyl \\
\hline & 10.521 & 10.548 & 1.0026 & 0.3 & $\mathrm{CE}$ \\
\hline & 12.069 & 12.236 & 1.0138 & 1.2 & CM 3.5 \\
\hline \multirow{3}{*}{ Methiopropamine } & 11.068 & 11.254 & 1.0168 & 1.4 & Succinyl \\
\hline & 11.943 & 12.116 & 1.0145 & 1.2 & $\mathrm{CE}$ \\
\hline & 13.832 & 14.098 & 1.0192 & 1.4 & CM 3.5 \\
\hline \multirow{3}{*}{ Thiopropamine } & 8.824 & 8.922 & 1.0111 & 0.5 & Succinyl \\
\hline & 11.397 & 11.493 & 1.0084 & 0.7 & \\
\hline & 13.056 & 13.281 & 1.0172 & 1.6 & CM 3.5 \\
\hline Other Chiral Substc & & & & & \\
\hline & 15.076 & 15.166 & 1.0060 & 0.6 & Succinyl \\
\hline $5-\mathrm{APi}$ & 17.973 & 18.515 & 1.0302 & 2.4 & $\mathrm{CE}$ \\
\hline & 25.210 & 26.355 & 1.0454 & 3.3 & CM 3.5 \\
\hline & 10.402 & 10.63 & 1.0219 & 1.2 & Succinyl \\
\hline MTTA & 14.094 & 14.230 & 1.0096 & 0.9 & $\mathrm{CE}$ \\
\hline & 18.003 & 18.247 & 1.0136 & 1.1 & CM 3.5 \\
\hline & 5.179 & n.d. & - & - & Succinyl \\
\hline MDAT & 6.797 & n.d. & - & - & $\mathrm{CE}$ \\
\hline & 7.366 & n.d. & - & - & CM 3.5 \\
\hline & 15.038 & n.d. & - & - & Succinyl \\
\hline EFLEA & 22.512 & n.d. & - & - & $\mathrm{CE}$ \\
\hline & 30.731 & n.d. & - & - & CM 3.5 \\
\hline
\end{tabular}

Note: Results of chiral separation attempts using succinyl-, carboxyethyl-, and carboxymethyl- $\beta$-CD, degree of substitution each 3.5 , n.d. $=$ not detected 
Table 2.7 Results of 136 investigated NPS

\begin{tabular}{|c|c|c|c|c|c|}
\hline & $\mathrm{t} 1(\min )$ & $\mathrm{t} 2(\mathrm{~min})$ & $\alpha(\mathrm{t} 2 / \mathrm{t} 1)$ & Rs & Selector \\
\hline \multicolumn{6}{|l|}{ Methylenedioxycathinones } \\
\hline & 11.924 & n.d. & - & - & Succinyl \\
\hline \multirow[t]{2}{*}{ Methylone } & 19.753 & 20.032 & 1.0141 & 0.9 & $\mathrm{CE}$ \\
\hline & 22.201 & 22.615 & 1.0186 & 1.3 & CM 3.5 \\
\hline \multirow{3}{*}{ 2-AIMP (5-MeO-Methylone) } & 12.540 & n.d. & - & - & Succinyl \\
\hline & 16.428 & 16.533 & 1.0064 & 0.7 & $\mathrm{CE}$ \\
\hline & 23.383 & 24.374 & 1.0424 & 3.3 & CM 3.5 \\
\hline \multirow{3}{*}{ Dimethylone } & 12.317 & n.d. & - & - & Succinyl \\
\hline & 14.520 & 14.641 & 1.0083 & 0.8 & $\mathrm{CE}$ \\
\hline & 18.297 & 18.502 & 1.0112 & 0.9 & CM 3.5 \\
\hline \multirow{3}{*}{ Butylone } & 13.725 & 14.206 & 1.0350 & 2.5 & Succinyl \\
\hline & 16.805 & n.d. & - & - & $\mathrm{CE}$ \\
\hline & 20.994 & n.d. & - & - & CM 3.5 \\
\hline \multirow{3}{*}{ N,N-Dimethylbutylone } & 14.374 & 14.715 & 1.0237 & 1.6 & Succinyl \\
\hline & 16.839 & n.d. & - & - & $\mathrm{CE}$ \\
\hline & 21.916 & n.d. & - & - & CM 3.5 \\
\hline \multirow{3}{*}{ Pentylone } & 13.205 & 13.472 & 1.0202 & 1.3 & Succinyl \\
\hline & 16.072 & 16.325 & 1.0157 & 1.5 & \\
\hline & 20.732 & 21.222 & 1.0236 & 2.1 & CM 3.5 \\
\hline \multicolumn{6}{|l|}{ Methylenedioxypyrovalerones } \\
\hline \multirow{3}{*}{ MDPV } & 14.046 & 14.347 & 1.0214 & 2.3 & Succinyl \\
\hline & 19.483 & 19.941 & 1.0235 & 4.0 & $\mathrm{CE}$ \\
\hline & 24.587 & 25.277 & 1.0281 & 4.2 & CM 3.5 \\
\hline \multirow{3}{*}{ MDPHP (3,4-MD-PHP) } & 9.953 & 10.143 & 1.0191 & 0.9 & Succinyl \\
\hline & 14.295 & n.d & - & - & \\
\hline & 17.036 & n.d & - & - & CM 3.5 \\
\hline \multirow{3}{*}{ MDPEP (MD-PV8) } & 12.120 & 12.293 & 1.0143 & 1.0 & Succinyl \\
\hline & 15.076 & 15.569 & 1.0327 & 2.7 & $\mathrm{CE}$ \\
\hline & 17.664 & 18.345 & 1.0386 & 3.5 & CM 3.5 \\
\hline \multicolumn{6}{|l|}{ Other Methylenedioxycathinones } \\
\hline \multirow{3}{*}{ N-Benzylnorbutylone } & 22.245 & 22.583 & 1.0152 & 1.1 & Succinyl \\
\hline & 29.716 & 30.282 & 1.0190 & 1.4 & \\
\hline & 29.461 & 30.041 & 1.0197 & 1.3 & CM 3.5 \\
\hline \multirow{3}{*}{ MDPT } & 11.622 & 11.884 & 1.0225 & 1.4 & Succinyl \\
\hline & 14.365 & n.d. & - & - & $\mathrm{CE}$ \\
\hline & 17.512 & n.d. & - & - & CM 3.5 \\
\hline \multicolumn{6}{|l|}{ Other Cathinones } \\
\hline \multirow{3}{*}{ bk-iVP } & 15.912 & n.d. & - & - & Succinyl \\
\hline & 23.258 & 23.480 & 1.0095 & 0.9 & \\
\hline & 28.491 & 28.972 & 1.0169 & 1.5 & CM 3.5 \\
\hline \multirow{3}{*}{ 5-PPDi } & 16.028 & n.d. & - & - & Succinyl \\
\hline & 22.722 & n.d. & - & - & $\mathrm{CE}$ \\
\hline & 27.685 & n.d. & - & - & CM 3.5 \\
\hline \multirow{3}{*}{ 5-BPDi } & 16.052 & n.d. & - & - & Succinyl \\
\hline & 22.305 & n.d. & - & - & $\mathrm{CE}$ \\
\hline & 26.285 & n.d. & - & - & CM 3.5 \\
\hline
\end{tabular}

Note: Results of chiral separation attempts using succinyl-, carboxyethyl-, and carboxymethyl- $\beta$-CD, degree of substitution each 3.5 , n.d. $=$ not detected

Table 3 Total number of chiral separations using each cyclodextrin

\begin{tabular}{lccccccc}
\hline & Native & Acetyl & HP & CM 0.5 & CM 3.5 & CE & Succinyl \\
\hline Baseline separation $(\mathrm{RS} \geq 2)$ & 6 & 13 & 6 & 47 & 28 & 13 & 22 \\
Separation (RS $<2)$ & 74 & 55 & 75 & 59 & 69 & 81 & 75 \\
Separation total & 80 & 68 & 81 & 106 & 97 & 94 & 97 \\
\hline No separation & 56 & 68 & 55 & 30 & 39 & 42 & 39 \\
Measurements total & 136 & 136 & 136 & 136 & 136 & 136 & 136 \\
\hline
\end{tabular}

Note: Comparison of Rs values of investigated substances with native-, acetyl-, hydroxypropyl-, carboxymethyl- (DS 0.5 and 3.5), carboxyethyl- and succinyl- $\beta$-cyclodextrin.

\subsection{Separation of positional isomers}

A further look was also taken on the separation of positional isomers with the set of negatively charged cyclodextrins, since distinction of positional isomers by classic achiral chromatography 
can be a difficult task. Simple amphetamines or cathinones monosubstituted at their phenyl ring were mainly traded first as para, later as meta and ortho form. They exist as two enantiomers each, leading to the conclusion that $e . g$. methylmethcathinone is present in six different forms. In this context, 17 substances were available as para and/or meta and/or ortho isomers. Successful separations of isomers as well as their isomeric migration order (IMO) is given in Table 4.

Table 4 Separation of positional isomers and their isomeric migration order (IMO)

\begin{tabular}{|c|c|c|c|c|c|c|}
\hline & \multicolumn{2}{|c|}{ CM 3.5} & \multicolumn{2}{|c|}{ CE 3.5} & \multicolumn{2}{|c|}{ Succinyl 3.5} \\
\hline & IMO & separ. & IMO & separ. & IMO & separ. \\
\hline \multicolumn{7}{|l|}{ Amphetamine } \\
\hline $2-/ 3-/ 4-F A$ & $3 / 2 / 1$ & bs & $2 / 1 / 3$ & o & $2 / 3 / 1$ & po \\
\hline 3,4-/2,5-DiMeO-A & $1 / 2$ & bs & $1 / 2$ & bs & $1 / 2$ & bs \\
\hline 2-/3-/4-FMA & $3 / 1 / 2$ & po & $2 / 3 / 1$ & bs & $2 / 3 / 1$ & bs \\
\hline 3,4-/2,3-MDMA & $2 / 1$ & bs & $2 / 1$ & bs & $2 / 1$ & bs \\
\hline 2-/3-/4-FEA & $3 / 2 / 1$ & po & $3 / 2 / 1$ & bs & $2 / 3 / 1$ & po \\
\hline \multicolumn{7}{|l|}{ Benzofuranes } \\
\hline 4-/5-/6-APB & $1 / 3 / 2$ & bs & $1 / 2 / 3$ & bs & $1 / 2 / 3$ & bs \\
\hline 5-/6-APDB & $1 / 2$ & bs & $1 / 2$ & bs & $1 / 2$ & bs \\
\hline 5-/6-EAPB & $1 / 2$ & bs & $1 / 2$ & bs & $1 / 2$ & bs \\
\hline \multicolumn{7}{|l|}{ Cathinones } \\
\hline 2-/3-/4-MMC & $1 / 2 / 3$ & bs & $1 / 2 / 3$ & bs & $1 / 2 / 3$ & bs \\
\hline 3,4-/2,4-DMMC & $2 / 1$ & bs & $2 / 1$ & bs & $2 / 1$ & bs \\
\hline 2-/3-/4-MeO-MC & $1 / 2 / 3$ & bs & $1 / 3 / 2$ & bs & $1 / 3 / 2$ & bs \\
\hline 2-/3-/4-CMC & $3 / 1 / 2$ & po & n.d./1/2 & po & $3 / 1 / 2$ & po \\
\hline 3-/4-EMC & $1 / 2$ & bs & $1 / 2$ & bs & $1 / 2$ & bs \\
\hline 2-/3-/4-FMC & $3 / 2 / 1$ & bs & $3 / 2 / 1$ & bs & $1 / 3 / 2$ & bs \\
\hline 3-/4-CEC & $1 / 2$ & o & $2 / 1$ & o & n.d./n.d. & o \\
\hline 3-/4-MEC & $1 / 2$ & bs & $1 / 2$ & bs & $1 / 2$ & bs \\
\hline 2-/3-/4-EEC & $1 / 3 / 2$ & bs & $1 / 3 / 2$ & bs & $1 / 3 / 2$ & bs \\
\hline
\end{tabular}

Note: $\mathrm{bs}=$ baseline separation, $\mathrm{po}=$ partial overlapping, $\mathrm{o}=$ overlapping, $\mathrm{n} . \mathrm{d} .=$ not detected

Except for mixtures of CMC (chloromethcathinone) and CEC (chloroethcathinone), baseline separations of the isomers were achieved with at least one of the used cyclodextrins. Most of the mixtures were separated with each negatively charged cyclodextrin. Though some were only partially separated, their migration order can be recognized and used for identification of positional isomers. Figure 2 shows separation and IMO of 2-EEC, 3-EEC and 4-EEC by means of carboxyethyl- $\beta$-cyclodextrin within $18 \mathrm{~min}$.

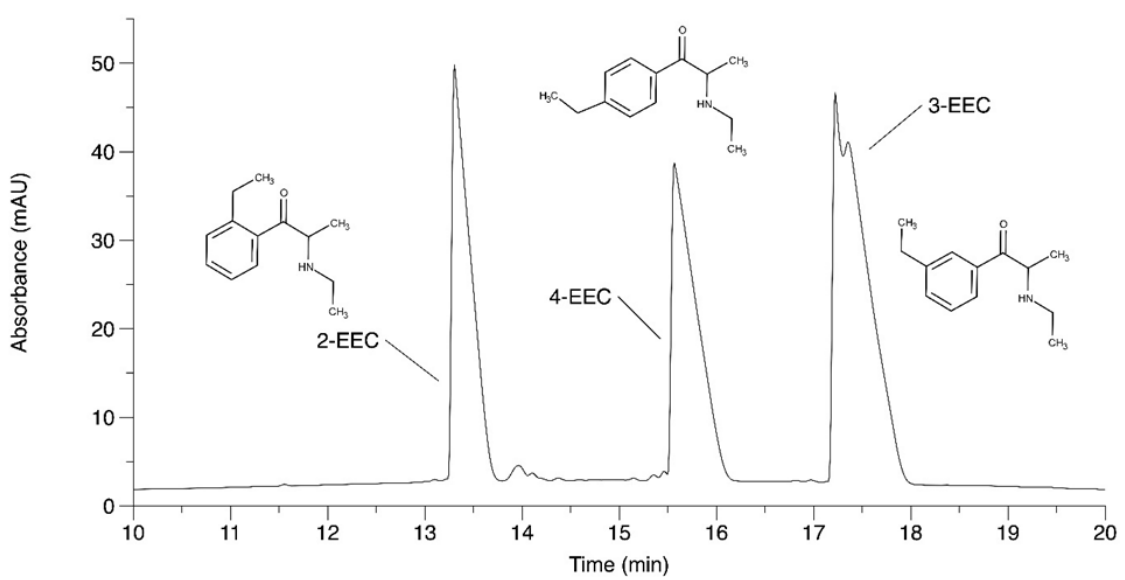

Figure 2 Isomeric migration order of ethylethcathinones, Conditions: $10 \mathrm{mM}$ di-sodium hydrogen phosphate; $10 \mathrm{mM}$ carboxyethyl- $\beta$-cyclodextrin; pH 2.5 ; UV $209 \mathrm{~nm}$; $+22 \mathrm{kV}$; Inj.: $10 \mathrm{mbar} / 5 \mathrm{sec}$

\subsection{Analysis of real-life samples}

To prove the usefulness of chiral separation of NPS with cyclodextrins, three different reallife samples were investigated using the presented method and CM-, CE- and succinyl- $\beta$-CD as chiral selectors. 
Sample A was a small amount of Crystal meth with an unknown chiral status found with an addicted patient in an Austrian hospital. First, sample A was measured; in contrary to the mostly commercially available racemic mixtures of NPS only one peak was observed and compared with the migration time of D-methamphetamine of a commercially available standard. Also the mixture of both samples revealed one peak, leading to the conclusion that sample A represents D-methamphetamine, which is identical with the eutomer S-(+)-methamphetamine. Additionally, each of the samples was mixed with the standard racemic methamphetamine to determine the enantiomeric migration order. Figure 3 shows an appropriate electropherogram with D-methamphetamine migrating after L-methamphetamine.

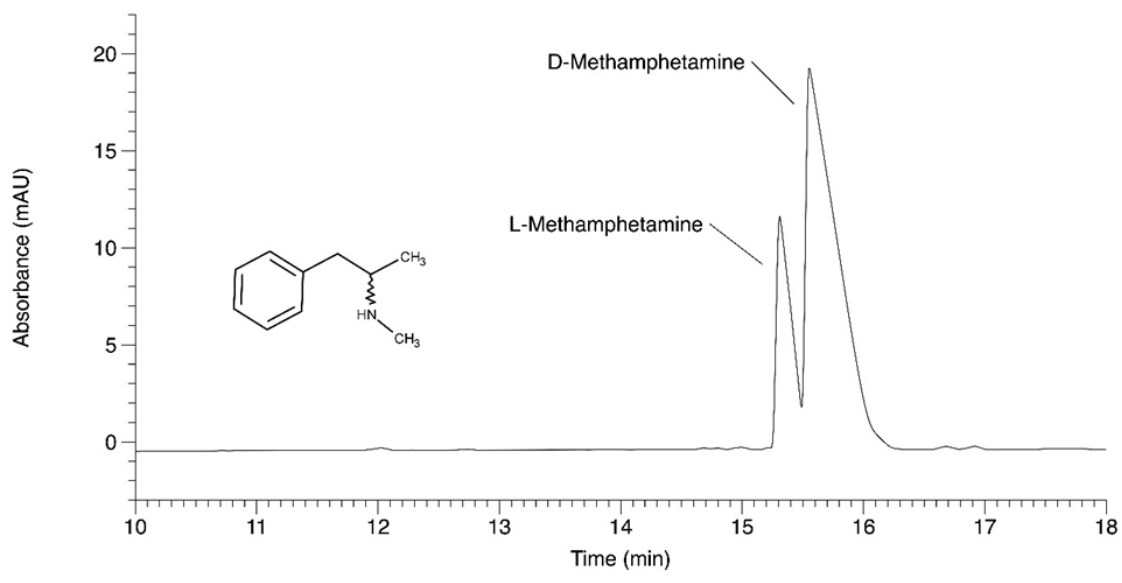

Figure 3 Determination of the enantiomeric status of a methamphetamine containing real-life sample. Mixture of commercially available racemic N-methamphetamine with a methamphetamine real-life sample; Conditions: $10 \mathrm{mM}$ di-sodium hydrogen phosphate; $10 \mathrm{mM}$ carboxymethyl- $\beta$-cyclodextrin; pH 2.5; UV 209 nm; +22 kV; Inj.: 10 mbar/5 sec

Sample B was expected to be a crystalline Ecstasy powder seized by police containing methylenedioxymethamphetamine (MDMA). Migration times of the sample, which was present as racemic mixture fit to those of a MDMA standard. A mixture of Sample B and MDMA standard showed no further peaks.

Sample C was assumed to be methylmethcathinone (MMC) of unknown isomeric status. Measurements of sample $\mathrm{C}$ showed similar migration times to 4-MMC. To make sure, sample $\mathrm{C}$ was added to a mixture of 2-, 3-, and 4-MMC with the expectation of one MMC peak to be spiked. As a result, Figure 4 shows no fit to the MMC peaks but an additional peak in the electropherogram. Therefore, sample $\mathrm{C}$ was a different substance.

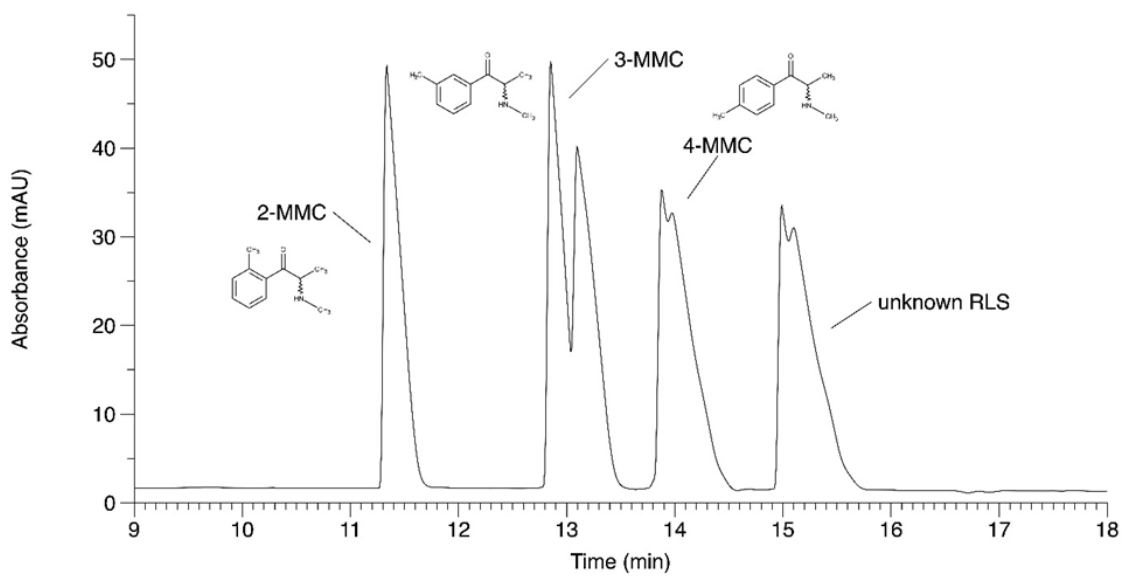

Figure 4 Separation of MMC isomers and an unidentified real-life sample. Mixture of 2-, 3- and 4-MMC with an unidentified real-life sample (RLS); Conditions: $10 \mathrm{mM}$ di-sodium hydrogen phosphate; $10 \mathrm{mM}$ carboxyethyl- $\beta$-cyclodextrin; $\mathrm{pH} 2.5$; UV $209 \mathrm{~nm}$; $+22 \mathrm{kV}$; Inj.: $10 \mathrm{mbar} / 5 \mathrm{sec}$

In addition, identity of sample A and B was confirmed by mass spectroscopy, and sam- 
ple $\mathrm{C}$ was identified as 3-methylethcathinone (3-MEC) by mass spectroscopy and infrared spectroscopy.

\subsection{Validation data}

To prove the robustness of the described method using the negatively charged cyclodextrins, intra- and interday validation was performed, with acceptable values for each chiral selector. As in work of Hägele et al. [42,43] pentedrone was chosen as model compound. A summary of the validation data with inter- and intraday measurements $n=5$ for each cyclodextrin is given in Table 5 .

Table 5 Summary of inter- and intraday validation

\begin{tabular}{llcccccc}
\hline & & t1 & RSD $\%$ & t2 & RSD & Rs & RSD \\
\hline \multirow{5}{*}{ Intraday validation } & CM- $\beta$-CD 3.5 & $16.59 \pm 0.54$ & 3.3 & $17.39 \pm 0.59$ & 3.4 & $4.2 \pm 0.4$ & 9.3 \\
& Succinyl- $\beta$-CD & $11.74 \pm 0.08$ & 0.7 & $11.74 \pm 0.07$ & 0.6 & $0.9 \pm 0.0$ & 0.0 \\
& CE- $\beta$-CD & $13.85 \pm 0.88$ & 6.4 & $14.36 \pm 0.95$ & 6.6 & $2.7 \pm 0.2$ & 6.1 \\
\hline \multirow{5}{*}{ Interday validation } & CM- $\beta$-CD 3.5 & $16.80 \pm 0.74$ & 4.4 & $17.68 \pm 0.82$ & 4.7 & $4.7 \pm 0.3$ & 5.9 \\
& Succinyl- $\beta$-CD & $11.50 \pm 1.04$ & 9.0 & $11.66 \pm 1.05$ & 9.0 & $1.0 \pm 0.1$ & 8.0 \\
& CE- $\beta$-CD & $13.92 \pm 0.11$ & 0.8 & $14.43 \pm 0.12$ & 0.9 & $2.7 \pm 0.1$ & 3.0 \\
\hline
\end{tabular}

\section{Conclusion}

The present work has shown the ability of $\beta$-cyclodextrins for enantioseparation of a huge number of NPS out of different substance classes. There is a clear trend towards the negatively charged CDs like carboxymethyl-, carboxyethyl-, and succinyl- $\beta$-cyclodextrin to be superior.

The quick and easy setup of buffer and samples, moderate retention times as well as the usage of common fused silica capillaries promote capillary electrophoresis to a promising alternative for chiral separations. In contrary to HPLC and GC methods, no special chiral columns are necessary, and the chiral selector is low in consumption and is added to the background electrolyte. It can be regarded as a resource saving and environmentally friendly approach because small amounts of solvents, buffer chemicals and analytes are used.

It turned out that NPS are traded as racemic mixtures, only the classic drug Crystal meth represents an exception. Most NPS are traded as hydrochloric salts, which requires no further sample preparation apart of dissolution in water.

In terms of structure elucidation, this method also helps to differ between positional isomers as an additional benefit in contrast to other identification methods. Moreover, the approach was found to be suitable for the investigation of real-life samples seized by police or collected in hospitals.

One simple method turned out to be sufficient for all presented measurements. Separations can be compared easily with respect to choose the optimal selector. Because of the versatile usability of this method, it might be useful for further upcoming NPS in future, both in terms of enantiomeric status as well as distinction of positional isomers.

\section{Conflict of interest}

The authors declare no conflict of interest.

\section{References}

[1] Schmid MG. Optical Detection of NPS Internet Products via HPLC-DAD Systems: A Selective Review, in Light in Forensic Science: Issues and Applications. Royal Society of Chemistry, 2018, 301-332.

https://doi.org/10.1039/9781788010344-00301

[2] EMCDDA. European Drug Report 2021, viewed December 22, 2021. https://doi.org/10.2810/18539

[3] United Nations 2013. The International Drug Control Conventions, viewed December 22, 2021 https://www.unodc.org/documents/commissions/CND/Int_Drug_Control_Conventions/Ebook/ The_International_Drug_Control_Conventions_E.pdf

[4] United Nations 1961. Drug Convention 1961, viewed December 22, 2021. https://undocs.org/en/ST/CND/1/Add.1/Rev.8 
[5] United Nations 1972. "Psychotropic Convention 1972." viewed December 22, 2021. https://undocs.org/ST/CND/1/Add.2/Rev.7

[6] Zhang YY, Li L, Ma AD, et al. Research Progress on Chiral Separation Methods and Toxic Effects of Methamphetamine Enantiomers. Fa Yi Xue Za Zhi, 2019, 35(3): 337-343. https://doi.org/10.12116/j.issn.1004-5619.2019.03.015

[7] Nishimura T, Takahata K, Kosugi Y, et al. Psychomotor effect differences between 1-methamphetamine and d-methamphetamine are independent of murine plasma and brain pharmacokinetics profiles. Journal of Neural Transmission, 2017, 124(5): 519-523. https://doi.org/10.1007/s00702-017-1694-y

[8] Jelen LA, Young AH and Stone JM. Ketamine: A tale of two enantiomers. Journal of Psychopharmacology, 2021, 35(2): 109-123. https://doi.org/10.1177/0269881120959644

[9] Ferris RM and Tang FL. Comparison of the effects of the isomers of amphetamine, methylphenidate and deoxypipradrol on the uptake of $1-[3 \mathrm{H}]$ norepinephrine and $[3 \mathrm{H}]$ dopamine by synaptic vesicles from rat whole brain, striatum and hypothalamus. The Journal of pharmacology and experimental therapeutics, 1979, 210(3): 422-428.

[10] Silva B, Palmeira A, Silva R, et al. S-(+)-Pentedrone and R-(+)-methylone as the most oxidative and cytotoxic enantiomers to dopaminergic SH-SY5Y cells: Role of MRP1 and P-gp in cathinones enantioselectivity. Toxicology and Applied Pharmacology, 2021, 416: 115442. https://doi.org/10.1016/j.taap.2021.115442

[11] Losacker M, Toennes SW, de Sousa Fernandes Perna EB, et al. Chiral Serum Pharmacokinetics of 4-Fluoroamphetamine after Controlled Oral Administration: Can (R)/(S)-Concentration Ratios Help in Interpreting Forensic Cases? Journal of Analytical Toxicology, 2021, 45(9): 985-992. https://doi.org/10.1093/jat/bkaa156

[12] Philogene-Khalid HL, Hicks C, Reitz AB, et al. Synthetic cathinones and stereochemistry: S enantiomer of mephedrone reduces anxiety- and depressant-like effects in cocaine- or MDPV-abstinent rats. Drug and Alcohol Dependence, 2017, 178: 119-125. https://doi.org/10.1016/j.drugalcdep.2017.04.024

[13] Curry DW, Young MB, Tran AN, et al. Separating the agony from ecstasy: R(-)-3,4methylenedioxymethamphetamine has prosocial and therapeutic-like effects without signs of neurotoxicity in mice. Neuropharmacology, 2018, 128: 196-206. https://doi.org/10.1016/j.neuropharm.2017.10.003

[14] Pitts EG, Curry DW, Hampshire KN, et al. ( \pm )-MDMA and its enantiomers: potential therapeutic advantages of R(-)-MDMA. Psychopharmacology, 2018, 235(2): 377-392. https://doi.org/10.1007/s00213-017-4812-5

[15] Botanas CJ, Perez Custodio RJ, Kim HJ, et al. R-methoxetamine exerts rapid and sustained antidepressant effects and fewer behavioral side effects relative to $S(+)$-methoxetamine. Neuropharmacology, 2021, 193: 108619 https://doi.org/10.1016/j.neuropharm.2021.108619

[16] Weiß JA, Pertl C and Schmid MG. Investigation of Two Seized Crystal Meth Labs in Austria - Parameters and Chirality Aspects of Methamphetamine Low Scale Synthesis. ARC Journal of Forensic Science, 2018, 3(1): 1-8. https://doi.org/10.20431/2456-0049.0301001

[17] Losacker M, Zörntlein S, Schwarze B, et al. Determination of the enantiomeric composition of amphetamine, methamphetamine and 3,4-methylendioxy-N-methylamphetamine (MDMA) in seized street drug samples from southern Germany. Drug Testing and Analysis, 2021, 3118. https://doi.org/10.1002/dta.3118

[18] Schmid MG and Hägele JS. Separation of enantiomers and positional isomers of novel psychoactive substances in solid samples by chromatographic and electrophoretic techniques - A selective review. Journal of Chromatography A, 2020, 1624. https://doi.org/10.1016/j.chroma.2020.461256

[19] Koster N, Clark CP and Kohler I. Past, present, and future developments in enantioselective analysis using capillary electromigration techniques. Electrophoresis, 2021, 42(1-2): 38-57. https://doi.org/10.1002/elps.202000151

[20] Hancu G, Papp LA, Tóth G, et al. The Use of Dual Cyclodextrin Chiral Selector Systems in the Enantioseparation of Pharmaceuticals by Capillary Electrophoresis: An Overview. Molecules, 2021, 26(8): 2261 . https://doi.org/10.3390/molecules26082261

[21] Hancu G, Orlandini S, Papp LA, et al. Application of Experimental Design Methodologies in the Enantioseparation of Pharmaceuticals by Capillary Electrophoresis: A Review. Molecules, 2021, 26(15): 4681. https://doi.org/10.3390/molecules26154681

[22] Peluso P and Chankvetadze B. Native and substituted cyclodextrins as chiral selectors for capillary electrophoresis enantioseparations: Structures, features, application, and molecular modeling. Electrophoresis, 2021, 42(17-18): 1676-1708. https://doi.org/10.1002/elps.202100053

[23] Lurie IS, Klein RFX, Dal Cason TA, et al. Chiral Resolution of Cationic Drugs of Forensic Interest by Capillary Electrophoresis with Mixtures of Neutral and Anionic Cyclodextrins. Analytical Chemistry, 1994, 66(22): 4019-4026. https://doi.org/10.1021/ac00094a026 
[24] Chinaka S, Tanaka S, Takayama N, et al. Simultaneous chiral analysis of methamphetamine and related compounds by capillary electrophoresis. Journal of Chromatography B: Biomedical Sciences and Applications, 2000, 749(1): 111-118. https://doi.org/10.1016/S0378-4347(00)00392-3

[25] Lurie IS, Odeneal NG, McKibben TD, et al. Effects of various anionic chiral selectors on the capillary electrophoresis separation of chiral phenethylamines and achiral neutral impurities present in illicit methamphetamine. Electrophoresis, 1998, 19(16-17): 2918-2925. https://doi.org/10.1002/elps.1150191620

[26] Rudaz S, Geiser L, Souverain S, et al. Rapid stereoselective separations of amphetamine derivatives with highly sulfated $\gamma$-cyclodextrin. Electrophoresis, 2005, 26(20): 3910-3920. https://doi.org/10.1002/elps.200500177

[27] Lanz M, Brenneisen R and Thormann W. Enantioselective determination of 3,4-methylenedioxymethamphetamine and two of its metabolites in human urine by cyclodextrin-modified capillary zone electrophoresis. Electrophoresis, 1997, 18(6): 1035. https://doi.org/10.1002/elps.1150180628

[28] Huang YS, Liu JT, Lin LC, et al. Chiral separation of 3,4-methylenedioxymeth- amphetamine and related compounds in clandestine tablets and urine samples by capillary electrophoresis/fluorescence spectroscopy. Electrophoresis, 2003, 24(6): 1097-1104. https://doi.org/10.1002/elps.200390128

[29] Scarcella D, Tagliaro F, Turrina S, et al. Optimization of a simple method for the chiral separation of phenethylamines of forensic interest based on cyclodextrin complexation capillary electrophoresis and its preliminary application to the analysis of human urine and hair. Forensic Science International, 1997, 89(1-2): 33. https://doi.org/10.1016/S0379-0738(97)00108-4

[30] Iwata YT, Inoue H, Kuwayama K, et al. Forensic application of chiral separation of amphetamine-type stimulants to impurity analysis of seized methamphetamine by capillary electrophoresis. Forensic Science International, 2006, 161(2-3): 92-96. https://doi.org/10.1016/j.forsciint.2006.01.018

[31] Iio R, Chinaka S, Takayama N, et al. Simultaneous Chiral Analysis of Methamphetamine and Related Compounds by Capillary Electrophoresis/Mass Spectrometry Using Anionic Cyclodextrin. Analytical Sciences, 2005, 21(1): 15-19. https://doi.org/10.2116/analsci.21.15

[32] Iwata YT, Garcia A, Kanamori T, et al. The use of a highly sulfated cyclodextrin for the simultaneous chiral separation of amphetamine-type stimulants by capillary electrophoresis. Electrophoresis, 2002, 23(9): 1328-1334. https://doi.org/10.1002/1522-2683(200205)23:9〈1328::AID-ELPS1328〉3.0.CO;2-Z

[33] Tagliaro F, Manetto G, Bellini S, et al. Simultaneous chiral separation of 3,4methylenedioxymethamphetamine (MDMA), 3-4-methylenedioxyamphetamine (MDA), 3,4methylenedioxyethylamphetamine (MDE), ephedrine, amphetamine and methamphetamine by capillary electrophoresis in uncoated and coated capillaries with native $\beta$-cyclodextrin as the chiral selector: Preliminary application to the analysis of urine and hair. Electrophoresis, 1998, 19(1): 42-50. https://doi.org/10.1002/elps.1150190109

[34] Pascual-Caro S, Borrull F, Aguilar C, et al. Comparison of different chiral selectors for the enantiomeric determination of amphetamine-type substances in human urine by solid-phase extraction followed by capillary electrophoresis-tandem mass spectrometry. Electrophoresis, 2021. https://doi.org/10.1002/elps.202100231

[35] Burrai L, Nieddu M, Pirisi MA, et al. Enantiomeric Separation of 13 New Amphetamine-Like Designer Drugs by Capillary Electrophoresis, Using Modified Cyclodextrins. Chirality, 2013, 25(10): 617-621. https://doi.org/10.1002/chir.22185

[36] Mohr S, Pilaj S and Schmid MG. Chiral separation of cathinone derivatives used as recreational drugs by cyclodextrin-modified capillary electrophoresis. Electrophoresis, 2012, 33(11): 1624-1630. https://doi.org/10.1002/elps.201100570

[37] Li L and Lurie IS. Regioisomeric and enantiomeric analyses of 24 designer cathinones and phenethylamines using ultra high performance liquid chromatography and capillary electrophoresis with added cyclodextrins. Forensic Science International, 2015, 254: 148-157. https://doi.org/10.1016/j.forsciint.2015.06.026

[38] Merola G, Fu H, Tagliaro F, et al. Chiral separation of 12 cathinone analogs by cyclodextrin-assisted capillary electrophoresis with UV and mass spectrometry detection. Electrophoresis, 2014, 35(21-22): 3231-3241. https://doi.org/10.1002/elps.201400077

[39] Nowak PM, Olesek K, Woźniakiewicz M, et al. Simultaneous enantioseparation of methcathinone and two isomeric methylmethcathinones using capillary electrophoresis assisted by 2-hydroxyethyl$\beta$-cyclodextrin. Electrophoresis, 2018, 39(19): 2406-2409. https://doi.org/10.1002/elps.201800142

[40] Taschwer M, Hofer MG and Schmid MG. Enantioseparation of benzofurys and other novel psychoactive compounds by $\mathrm{CE}$ and sulfobutylether $\beta$-cyclodextrin as chiral selector added to the BGE. Electrophoresis, 2014, 35(19): 2793-2799. https://doi.org/10.1002/elps.201400164 
[41] Schutzner W and Fanali S. Enantiomers resolution in capillary zone electrophoresis by using cyclodextrins. Electrophoresis, 1992, 13(1): 687-690.

https://doi.org/10.1002/elps.11501301146

[42] Hägele JS, Hubner EM and Schmid MG. Determination of the chiral status of different novel psychoactive substance classes by capillary electrophoresis and $\beta$-cyclodextrin derivatives. Chirality, 2020, 32(9): 1191-1207.

https://doi.org/10.1002/chir.23268

[43] Hägele JS, Hubner EM and Schmid MG. Chiral separation of cathinone derivatives using $\beta$ cyclodextrin-assisted capillary electrophoresis-Comparison of four different $\beta$-cyclodextrin derivatives used as chiral selectors. Electrophoresis, 2019, 40(14): 1787-1794.

https://doi.org/10.1002/elps.201900085 